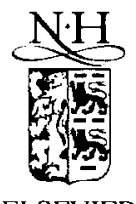

ELSEVIER

Astroparticle Physics 5 (1996) 1-26

Astroparticle

Physics

\title{
Neutralino dark matter in supersymmetric models with non-universal scalar mass terms
}

\author{
V. Berezinsky ${ }^{a, 1}$, A. Bottino ${ }^{b, c, 2}$, J. Ellis ${ }^{d, 3}$, N. Fornengo ${ }^{e, c, 4}$, G. Mignola ${ }^{b, c, 5}$, S. Scopel ${ }^{f, g, 6}$ \\ a INFN, Laboratori Nazionali del Gran Sasso, 67010 Assergi (AQ), Italy

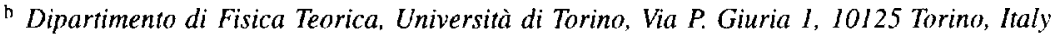 \\ c INFN. Sezione di Torino, Via P. Giuria 1, I0125 Torino, Italy \\ d Theoretical Physics Division. CERN, CH-1211 Geneva 23, Switzerland \\ ' Department of Physics and Astronomy, The Johns Hopkins University, Baltimore. MD 21218, USA \\ ' Dipartimento di Fisica, Università di Genova, Via Dodecaneso 33, 16146 Genova, Italy \\ INFN, Sezione di Genova, Via Dodecaneso 33, 16146 Genova, Italy
}

Received 9 August 1995

\begin{abstract}
Neutralino dark matter is studied in the context of a supergravity scheme where the soft scalar mass terms are not constrained by universality conditions at the grand unification scale. We analyse in detail the consequences of the relaxation of this universality assumption on the supersymmetric parameter space, on the neutralino relic abundance and on the event rate for the direct detection of relic neutralinos.
\end{abstract}

\section{Introduction}

The phenomenology of neutralino dark matter has been studied extensively in the Minimal Supersymmetric extension of the Standard Model (MSSM) [1]. This model incorporates the same gauge group as the Standard Model and the supersymmetric extension of its particle content. The Higgs sector is slightly modified as compared to that of the Standard Model: the MSSM requires two Higgs doublets $H_{1}$ and $H_{2}$ in order to give mass both to down- and up-type quarks and to cancel anomalies. After Electro-Weak Symmetry Breaking (EWSB), the physical Higgs fields consist of two charged particles and three neutral ones: two scalar fields ( $h$ and $H$ ) and one pseudoscalar $(A)$. The Higgs sector is specified at the tree level by two independent

\footnotetext{
'E-mail: berezinsky@Ings.infn.it.

${ }^{2}$ E-mail: bottino@to.infn.it.

${ }^{3}$ E-mail: johne@cernvm.cern.ch.

${ }^{4}$ E-mail: fornengo@jhup.pha.jhu.edu.

${ }^{5}$ E-mail: mignola@to.infn.it.

${ }^{6}$ E-mail: scopel@ge.infn.it.
} 


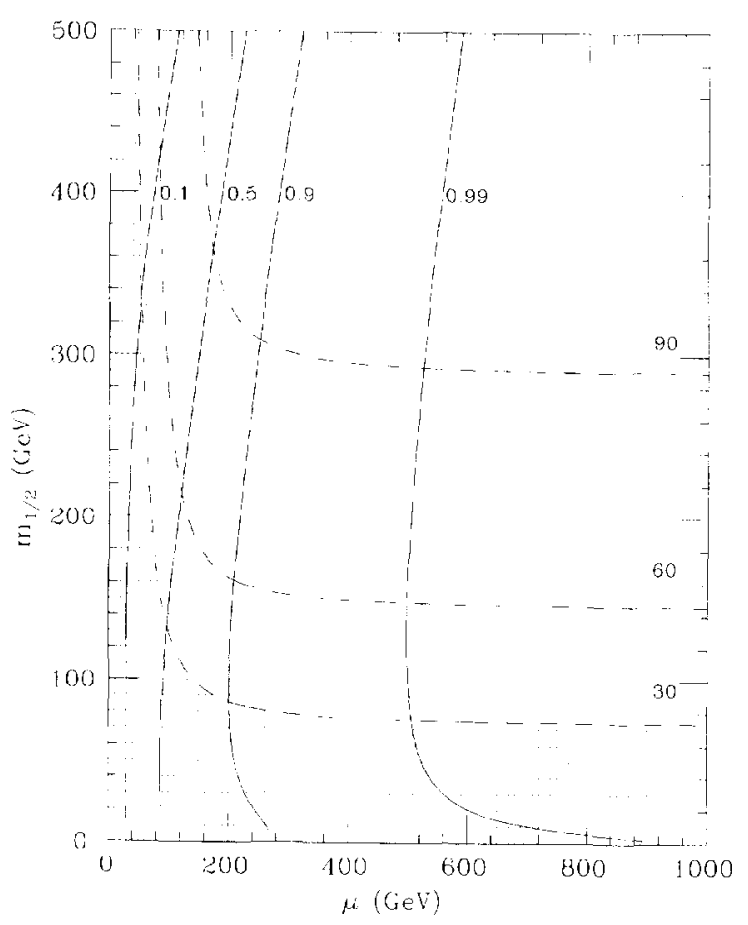

Fig. 1. The $\left(m_{1 / 2}, \mu\right)$ plane for $\tan \beta=8$. The lines of constant $m_{X}=30 \mathrm{GeV}, 60 \mathrm{GeV}, 90 \mathrm{GeV}$ are displayed as dashed lines. The lines of constant $\mathrm{P}=0.1,0.5,0.9,0.99$ are shown as solid lines. The dotted region denotes the domain forbidden by present LEP data.

parameters: the mass of one of the physical Higgs fields and the ratio of the two vacuum expectation values, usually defined as $\tan \beta=v_{2} / v_{1} \equiv\left\langle H_{2}\right\rangle /\left\langle H_{1}\right\rangle$. The supersymmetric sector of the model introduces some other free parameters: the mass parameters $M_{1}, M_{2}$ and $M_{3}$ for the supersymmetric partners of gauge fields (gauginos), the Higgs-mixing parameter $\mu$ and, in general, all the masses of the scalar partners of the fermions (sfermions).

In the MSSM it is generally assumed that the gaugino masses are equal at the grand unification scale $M_{G U T}$ : $M_{i}\left(M_{G U T}\right) \equiv m_{1 / 2}$ and hence are related at lower scales by

$$
M_{1}: M_{2}: M_{3}=\alpha_{1}: \alpha_{2}: \alpha_{3}
$$

where the $\alpha_{i}(i=1,2,3)$ are the coupling constants of the three Standard Model gauge groups. The neutralinos are mass-eigenstate linear superpositions of the two neutral gauginos $(\tilde{\gamma}$ and $\tilde{Z})$ and the two neutral higgsinos $\left(\tilde{H}_{1}\right.$ and $\left.\tilde{H}_{2}\right)$

$$
\chi=a_{1} \tilde{\gamma}+a_{2} \tilde{Z}+a_{3} \tilde{H}_{1}+a_{4} \tilde{H}_{2} .
$$

The neutralino sector depends, at the tree-level, on the following (low-energy) parameters: $M_{1}=\frac{5}{3} \tan ^{2} \theta_{W} M_{2}$, $M_{2} \simeq 0.8 m_{1 / 2}, \mu$ and $\tan \beta$. Neutralino properties are naturally discussed in the $\left(m_{1 / 2}, \mu\right)$ plane, for a fixed value of $\tan \beta$. As an example, in Fig. 1 the lines of constant mass for the lightest neutralino $\left(m_{\chi}\right)$ and constant gaugino fractional weight $\left(P=a_{1}^{2}+a_{2}^{2}\right)$ are plotted in the $\left(m_{1 / 2}, \mu\right)$ plane for $\tan \beta=8$. We observe that the mass of the lightest neutralino increases from the bottom left to the top right, while the neutralino composition changes from higgsino dominance in the top-left region of the plane to gaugino dominance in the hottom-right. The regions forbidden by accelerator data are also displayed in Fig. 1.

The low-energy MSSM scheme is a purely phenomenological approach, whose basic idea is to impose as few model-dependent restrictions as possible. In this approach the lightest neutralino is a favourite candidate for 
cold dark matter. This scheme has been employed extensively in the analysis of the size and the relevance of various possible signals of neutralino dark matter: direct detection [2-4], signals due to neutralino annihilation in celestial bodies, namely the Earth and the Sun [5,6], and signals from neutralino annihilation in the galactic halo [7]. The MSSM provides a useful framework in which neutralino phenomenology may be analysed without strong theoretical prejudices which could, a posteriori, turn out to be incorrect. This scheme is also frequently employed in analyses of the discovery potential of future accelerators [8].

At a more fundamental level, it is natural to implement this phenomenological scheme within the supergravity framework [9-11]. One attractive feature of the ensuing model is the connection between soft supersymmetry breaking and EWSB, which would then be induced radiatively. The essential elements of the model are described by a Yang-Mills Lagrangian, the superpotential, which contains all the Yukawa interactions between the standard and supersymmetric fields, and by the soft-breaking Lagrangian, which models the breaking of supersymmetry. Here we only recall the soft supersymmetry breaking terms

$$
\begin{aligned}
& -\mathcal{L}_{s o f f}=\sum_{i} m_{i}^{2}\left|\phi_{i}\right|^{2} \\
& \quad+\left\{\left[A_{a b}^{l} h_{a b}^{l} \tilde{L}_{a} H_{1} \tilde{R}_{h}+A_{a b}^{d} h_{a b}^{d} \tilde{Q}_{a} H_{1} \tilde{D}_{b}+A_{a b}^{u} h_{a b}^{u} \tilde{Q}_{a} H_{2} \tilde{U}_{b}+\text { h.c. }\right]-B \mu H_{1} H_{2}+\text { h.c. }\right\} \\
& \quad+\sum_{i} M_{i}\left(\lambda_{i} \lambda_{i}+\bar{\lambda}_{i} \bar{\lambda}_{i}\right)
\end{aligned}
$$

where the $\phi_{i}$ are the scalar fields, the $\lambda_{i}$ are the gaugino fields, $H_{1}$ and $H_{2}$ are the two Higgs fields, $\tilde{Q}$ and $\tilde{L}$ are the doublet squark and slepton fields, respectively, and $\tilde{U}, \tilde{D}$ and $\tilde{R}$ denote the $S U(2)$-singlet fields for the up-squarks, down-squarks and sleptons. In Eq. (3), $m_{i}$ and $M_{i}$ are the mass parameters of the scalar and gaugino fields, respectively, and $A$ and $B$ denote trilinear and bilinear supersymmetry breaking parameters, respectively. The Yukawa interactions are described by the parameters $h$, which arc related to the masses of the standard fermions by the usual expressions, e.g., $m_{t}=h^{i} v_{2}$.

The supergravity framework is usually implemented with a number of restrictive assumptions about unification at $M_{G U T}$ :

(i) Unification of the gaugino masses: $M_{i}\left(M_{G U T}\right) \equiv m_{1 / 2}$,

(ii) Universality of the scalar masses with a common mass denoted by $m_{0}: m_{i}\left(M_{G U T}\right) \equiv m_{0}$,

(iii) Universality of the trilinear scalar couplings: $A^{l}\left(M_{G U T}\right)=A^{d}\left(M_{G U T}\right)=A^{u}\left(M_{G U T}\right) \equiv A_{0} m_{0}$.

These conditions have strong consequences for low-energy supersymmetry phenomenology, and in particular for the properties of the neutralino as dark matter particle. Typically, the lightest neutralino is constrained to regions of gaugino dominance, that entail a large relic abundance (in wide regions of the parameter space $\Omega_{\chi} h^{2}$ exceeds the cosmological upper bound) and a small direct detection rate for neutralino dark matter. Indirect signals from the neutralino, such as high-energy neutrinos from the Earth and Sun, and the products of annihilation in the halo, are practically undetectable [11].

The above assumptions, particularly (ii) and (iii), are not very solid, since universality may occur at a scale higher than $M_{G U T}$, i.e., the Planck scale or string scale [12], in which case renormalization above $M_{G U T}$ may weak universality in the $m_{i}$, e.g., between scalars in $\underline{\overline{5}}$ and $\underline{10}$ representations of $S U(5)$ [13]. Moreover, in many string models the $m_{i}$ 's are not universal even at the string scale.

In a number of recent works [14,15], deviations from some of the unification conditions have been considered. In particular, in Ref. [14] phenomenological consequences for neutralinos of a relaxation of assumption (ii) have been analysed in the regime of large values of $\tan \beta$. It has been shown that deviations from condition (ii) may entail a changeover in neutralino composition from a gaugino-like state to a higgsino-like state (or at least to a higgsino-gaugino mixed state), with important consequences for neutralino phenomenology.

In this paper, we first explore, over the full range of $\tan \beta$, the various scenarios which may occur when condition (ii) is relaxed, with an approach which is similar to the one adopted in the large-tan $\beta$ analysis 
of Ref. [14]. We then discuss in detail the ensuing consequences for neutralino dark matter, with particular emphasis for its direct detection.

In the following, we first discuss which constraints can be applied to the parameters when specific physical requirements are imposed. In Section 2, we summarize the conditions implied by radiative EWSB and define the type of departure from universality examined in this paper. Then, in Section 3 we establish some upper bounds on the supergravity parameters by requiring that radiative EWSB does not occur with excessive fine tuning among different terms. In Sect.IV we analyse in detail the constraints due to the requirement that EWSB takes place radiatively. Subsequently, in Section 5 cosmological constraints, derived from the evaluation of the neutralino relic abundance, are discussed. Other constraints, from experimental data on $b \rightarrow s \gamma$ processes and on the mass of the bottom quark $m_{b}$, are applied in Section 6. In Section 7 the effects of these various constraints are first displayed in the $\left(m_{1 / 2}, m_{0}\right)$ plane for fixed $\tan \beta$ and $A_{0}$, and then shown in the $\left(m_{1 / 2}, \mu\right)$ plane, which provides the most useful representation for discussing neutralino phenomenology. We recall some specific properties of the neutral Higgs bosons in Section 8. Finally, in Section 9 event rates for direct detection of neutralino dark matter are discussed. Conclusions are presented in the last section.

\section{Radiative EWSB}

We recall that the tree-level scalar potential for the neutral Higgs fields may be written in the form

$$
V_{0}=\left(M_{H_{1}}^{2}+\mu^{2}\right)\left|H_{1}\right|^{2}+\left(M_{H_{2}}^{2}+\mu^{2}\right)\left|H_{2}\right|^{2}-B \mu\left(H_{1} H_{2}+\text { h.c. }\right)+\text { quartic D terms. }
$$

The parameters of this potential must obey the following physical conditions:

$$
\begin{aligned}
& \sin 2 \beta=\frac{-2 B \mu}{M_{H_{1}}^{2}+M_{H_{2}}^{2}+2 \mu^{2}}, \\
& M_{Z}^{2}=2 \frac{M_{H_{1}}^{2}-M_{H_{2}}^{2} \tan ^{2} \beta}{\tan ^{2} \beta-1}-2 \mu^{2}, \\
& M_{A}^{2}=M_{H_{1}}^{2}+M_{H_{2}}^{2}+2 \mu^{2}>0 .
\end{aligned}
$$

Here $M_{A}$ is the mass of the CP-odd neutral Higgs boson (see Section 8 below), and Eq. (7) must in fact be strengthened to $M_{A} \geq\left(M_{A}\right)_{l b}$, where $\left(M_{A}\right)_{l b}$ is the experimental lower bound [16]. For instance, for $\tan \beta \gtrsim 3,\left(M_{A}\right)_{b b} \simeq 45 \mathrm{GeV}$. Notice that the sign of $\mu$ is defined according to the convention of reference [1]. We remark that although Eqs. (4)-(7) are expressed at the tree level, in our actual calculations 1-loop corrections to $V_{0}$ [17] have been included. The $M_{H_{i}}$ 's (as well as the sfermion and the gaugino masses and the parameters $A, B$ and $\mu$ ) evolve from the $M_{G U T}$ scale down to the $M_{Z}$ scale according to the Renormalization Group Equations (RGEs). This is how Eq. (6) may be satisfied, even if $M_{H_{1}}$ and $M_{H_{2}}$ are equal at $M_{G U T}$.

In this work we consider deviations from universality in the scalar masses at $M_{G U T}$, which split $M_{H_{1}}$ from $M_{H_{2}}$. This effect is parameterized as

$$
M_{H_{i}}^{2}\left(M_{G U T}\right)=m_{0}^{2}\left(1+\delta_{i}\right)
$$

The parameters $\delta_{i}$ which quantify the departure from universality for the $M_{H_{i}}^{2}$ will be varied in the range $(-1,+1)$, but are taken to be independent of the supersymmetry parameters. This is an Ansatz, since, when evolving the scalar masses from the unification scale (Planck scale or string scale) to the GUT scale $M_{G U T}$, the deviation parameters are in general functions of all the supersymmetry parameters [18].

Following a common procedure, Eq. (5) is used to replace the parameter $B$ by $\tan \beta$. Thus the set of independent parameters becomes $m_{1 / 2}, m_{0}, A_{0}, \tan \beta$, and $\mu^{2}$ is given in terms of these parameters by Eq. (6), suitably corrected by 1-loop effects: only the sign of $\mu$ remains undetermined. Obviously, values of $\mu^{2}$ are 
accepted only if they exceed the experimental lower bound $\mu_{l b}^{2}$, which is derived from the lower limit on the chargino mass [16]: $\left|\mu_{l b}\right| \simeq 45 \mathrm{GeV}$.

We have solved the RGEs using the 1-loop beta functions including the whole supersymmetric particle spectrum from the GUT scale down to $M_{Z}$, neglecting the possible effects of intermediate thresholds. Two-loop and threshold effects on the running of the gauge and Yukawa couplings are known not to exceed $10 \%$ of the final result [19]. While this is of crucial importance as far as gauge coupling unification is concerned [19], it is a second-order effect on the evolution of the soft masses. Since neutralino properties are studied over a wide range of variation for the high scale parameters, such a degree of refinement is not required here.

In order to specify the supersymmetry phenomenology, boundary conditions for the gauge and Yukawa couplings have to be specified. Low-scale values for the gauge couplings and for the top-quark and the taulepton Yukawa couplings are fixed using present experimental results. In particular, we assign for the top mass the value $m_{t}=178 \mathrm{GeV}$ [20]. In addition, we require the unification of the bottom and tau Yukawa couplings at the GUT scale, as would be suggested by a unifying group that includes an $S U(5)$-like structure [21].

The values of $M_{H_{1}}$ and $M_{H_{2}}$ at the $M_{Z}$ scale, obtained from the RGEs, may be parameterized in the following way:

$$
M_{H_{i}}^{2}=a_{i} m_{\mathrm{1} / 2}^{2}+b_{i} m_{0}^{2}+c_{i} A_{0}^{2} m_{0}^{2}+d_{i} A_{0} m_{0} m_{1 / 2}
$$

(Notice that, in our notation, all running quantities written without any further specification are meant to denote their values at $M_{Z}$.) The coefficients in the expression (9) are functions of $\tan \beta$ and of the $\delta_{i}$ 's. They are displayed in Fig. 2 (a,b) for the case of universal scalar masses, (i.e., $\delta_{i}=0$ ). The cocfficients for $M_{H_{2}}^{2}$ turn out to be very stable as functions of $\tan \beta$, except for small $\tan \beta$. More precisely, $a_{2} \sim-2.5$ for $\tan \beta \gtrsim 4$ with all the other coefficients much smaller (of order 0.1). As far as $M_{H_{1}}^{2}$ is concerned, whereas $c_{1}$ and $d_{1}$ are again very stable (of order 0.1 ), $a_{1}$ and $b_{1}$ vary rapidly as functions of $\tan \beta$. This property of $a_{1}$ and $b_{1}$ is due to the very fast increase of $h^{b}$ for increasing $\tan \beta$.

When a departure from $m_{0}$ universality is introduced, the coefficients in Eq. (9), except for $a_{1}$ and $a_{2}$, become functions of the parameters $\delta_{i}: b_{1}, c_{1}$ and $d_{1}$ depend on $\delta_{1}$ and $b_{2}, c_{2}$ and $d_{2}$ on $\delta_{2}$. Whereas the $b_{i}$ 's are rapidly-increasing functions of the $\delta_{i}$ 's, the $c_{i}$ 's and the $d_{i}$ 's are rather insensitive to these parameters.

Stringent constraints on the parameters $m_{1 / 2}, m_{0}, A_{0}$ and $\tan \beta$ follow from the request that the $M_{H_{i}}^{2}$ 's, evaluated from Eq. (9), satisfy Eqs. (6)-(7). Explicitly, we require that $\mu^{2}$ and $M_{A}^{2}$, given by the expressions

$$
\begin{aligned}
\mu^{2} & =\frac{1}{\tan ^{2} \beta-1}\left\{\left(a_{1}-a_{2} \tan ^{2} \beta\right) m_{1 / 2}^{2}+\left(b_{1}-b_{2} \tan ^{2} \beta\right) m_{0}^{2}\right. \\
& \left.+\left(c_{1}-c_{2} \tan ^{2} \beta\right) A_{0}^{2} m_{0}^{2}+\left(d_{1}-d_{2} \tan ^{2} \beta\right) A_{0} m_{0} m_{1 / 2}\right\}-\frac{M_{Z}^{2}}{2} \\
& \equiv J_{1} m_{1 / 2}^{2}+J_{2} m_{0}^{2}+J_{3} A_{0}^{2} m_{0}^{2}+J_{4} A_{0} m_{0} m_{1 / 2}-\frac{M_{Z}^{2}}{2} \\
M_{A}^{2} & =\left(a_{1}+a_{2}+2 J_{1}\right) m_{1 / 2}^{2}+\left(b_{1}+b_{2}+2 J_{2}\right) m_{0}^{2} \\
& +\left(c_{1}+c_{2}+2 J_{3}\right) A_{0}^{2} m_{0}^{2}+\left(d_{1}+d_{2}+2 J_{4}\right) A_{0} m_{0} m_{1 / 2}-M_{Z}^{2} \\
& \equiv K_{1} m_{1 / 2}^{2}+K_{2} m_{0}^{2}+K_{3} A_{0}^{2} m_{0}^{2}+K_{4} A_{0} m_{0} m_{1 / 2}-M_{Z}^{2}
\end{aligned}
$$

satisfy the conditions: $\mu^{2} \geq \mu_{l b}^{2}, M_{A} \geq\left(M_{A}\right)_{l b}$ mentioned earlier.

The coefficients $J_{i}$ and $K_{i}$ in Eqs. (10), (11) are plotted as functions of $\tan \beta$ in Fig. 2 (c,d) for the case of $m_{0}$ universality. In Fig. $2 \mathrm{c}$ we notice that all the $J_{i}$ 's are positive, with $J_{1}$ dominating over the others: for $\tan \beta \gtrsim 4$, one has $J_{1} \simeq 2.4$. As far as the coefficients $K_{i}$ are concerned, we see in Fig. $2 \mathrm{~d}$ that only two of them are sizeable: $K_{1}$ and $K_{2}$. They are both decreasing functions of $\tan \beta$, with $K_{1}>K_{2}$. At very large 


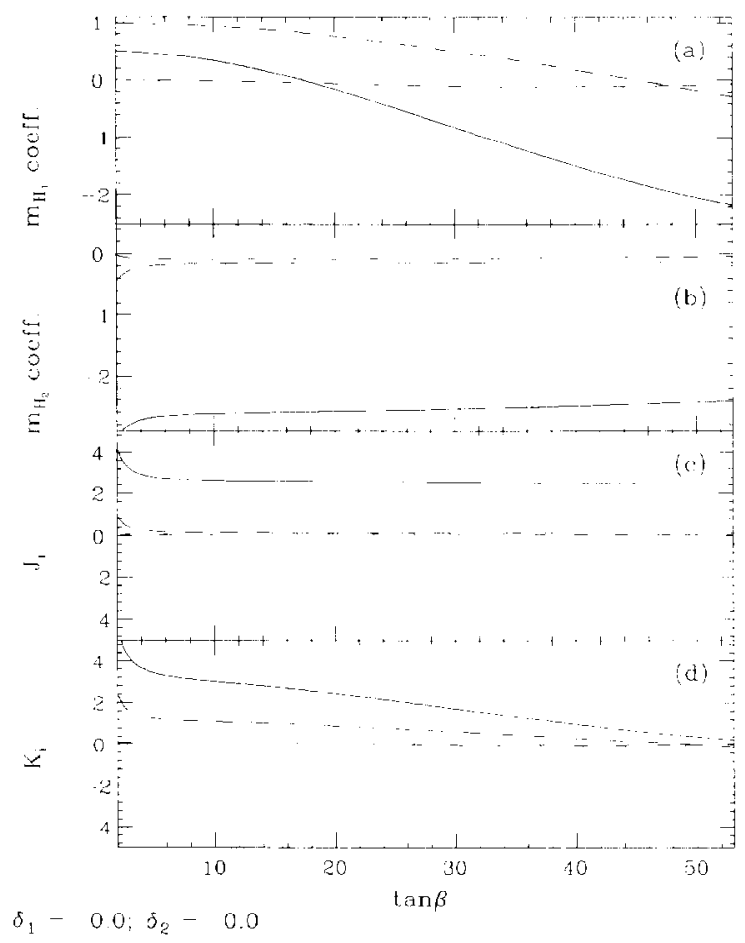

Fig. 2. Coefficients of the polynomial expressions (9)-(11) as functions of $\tan \beta$ : (a) coefficients of $M_{H_{1}}^{2}: a_{1}$ solid line. $b_{1}$ dashed line. $c_{1}$ dot-dashed line and $d_{1}$ dotted line, (b) coefficients of $M_{H_{2}}^{2}: a_{2}$ solid line, $b_{2}$ dashed line, $c_{2}$ dot-dashed line and $d_{2}$ dotted line. (c) coefficients of $\mu^{2}: J_{1}$ solid line, $J_{2}$ dashed line, $J_{3}$ dot-dashed line and $J_{4}$ dotted line, (d) coefficients of $M_{A}^{2}: K_{1}$ solid line, $K_{2}$ dashed line, $K_{3}$ dot-dashed line and $K_{4}$ dotted line.

$\tan \beta$ these coefficients become very small, and $K_{2}$ even becomes negative (but still small in magnitude) at $\tan \beta \gtrsim 50$.

In the case of non-universality, the coefficients $J_{i}$ and $K_{i}$, except for $J_{1}$ and $K_{1}$, become functions of the parameters $\delta_{i}$. We will see in Section 4 that many important features of the supersymmetry parameter space depend on the signs of the two coefficients $J_{2}$ and $K_{2}$. We show in Figs. 3 and 4 how their signs depend on the values of the $\delta_{i}$ 's. In Fig. 3 the lines $J_{2}=0$ are plotted in the $\left(\delta_{2}, \delta_{1}\right)$ plane for a few values of $\tan \beta$ : for each value of $\tan \beta, J_{2}$ is negative in the region above the relevant $J_{2}=0$ line and positive below. Similarly, in Fig. 4 the $K_{2}=0$ lines are displayed in the same $\left(\delta_{2}, \delta_{1}\right)$ plane at fixed $\tan \beta: K_{2}$ is negative above the $K_{2}=0$ lines, and positive below.

We now make a few comments related to Eq. (11), since the value of $M_{A}$ plays a very crucial role in a number of important neutralino properties. This is due to the fact that many physical processes involving neutralinos are mediated by the neutral Higgs bosons. Thus the value of $M_{A}$ determines the size of the relevant cross sections both through $M_{A}^{2}$-dependence in propagators and, in an implicit way, through the couplings of the $h$ and $H$ bosons to quarks and to the lightest neutralino $\chi$ (see Section 8). As a consequence, a small value of $M_{A}$ has the effect of enhancing the magnitude of the relevant cross sections.

What values of $M_{A}$ do we obtain from Eq. (11)? Because of the properties of the coefficients $K_{i}$ previously analysed, $M_{A}$ turns out to be a rapidly-decreasing function of $\tan \beta$. In Fig. 5, $M_{A}$ is displayed at the representative point $m_{0}=50 \mathrm{GeV}, m_{1 / 2}=200 \mathrm{GeV}$ ( 1 -loop corrections to $M_{A}$ have been included in the calculation). One notices that $M_{A}$ is $O\left(M_{Z}\right)$ for $\tan \beta \gtrsim 45$. This feature provides one of the most appealing scenarios for neutralino phenomenology. 

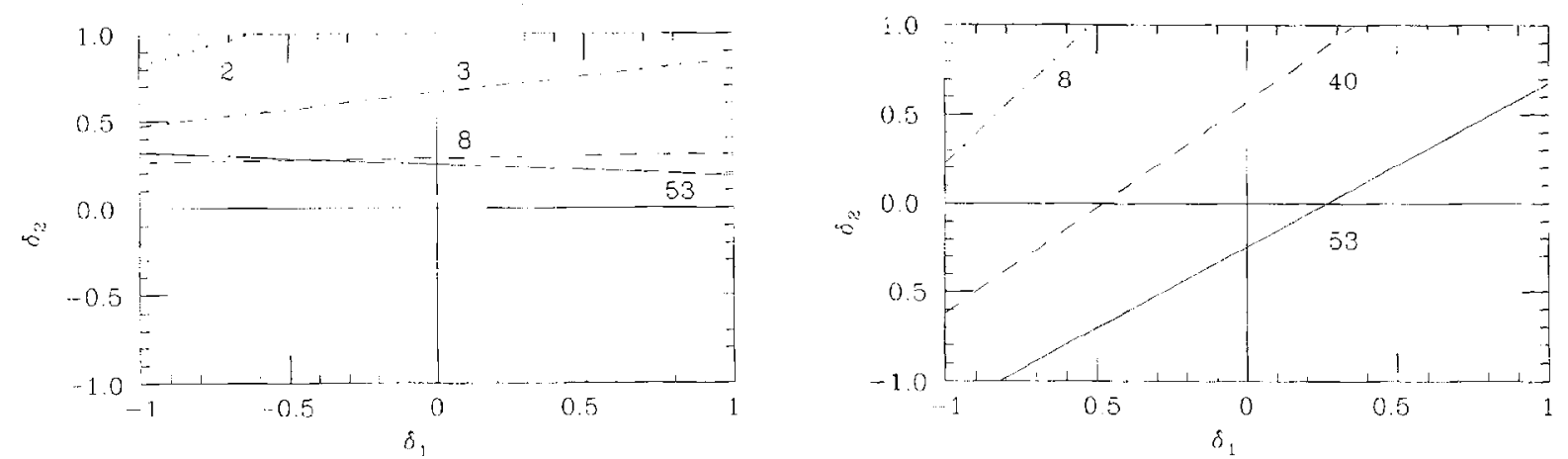

Fig. 3. In the $\left(\delta_{2}, \delta_{1}\right)$ plane, the lines where $J_{2}=0$ at fixed $\tan \beta$ are displayed: $\tan \beta=53$, solid line; $\tan \beta=8$, dashed line; $\tan \beta=3$, dot-dashed line; $\tan \beta=2$, dotted line.

Fig. 4. In the $\left(\delta_{2}, \delta_{1}\right)$ plane, the lines where $K_{2}=0$ at fixed $\tan \beta$ are displayed: $\tan \beta=53$, solid line; $\tan \beta=40$, dashed line: $\tan \beta=8$. dot-dashed line.

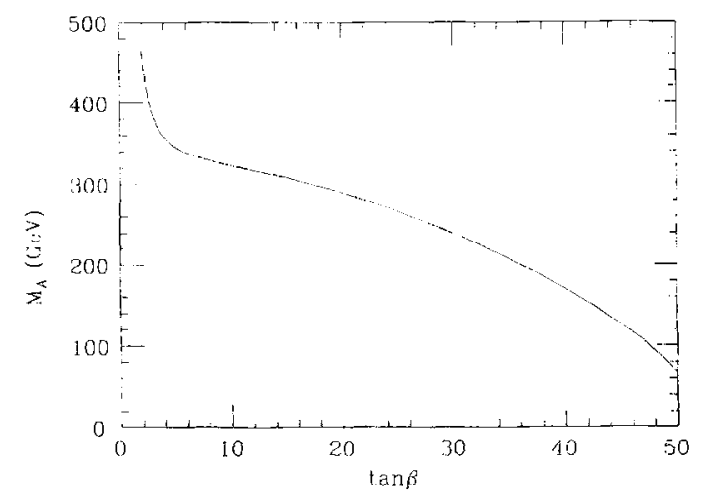

Fig. 5. Graph of $M_{A}$ as a function of $\tan \beta$ at the representative point $m_{11}=50 \mathrm{GeV}, m_{1 / 2}=200 \mathrm{GeV}$.

\section{Constraints due to the absence of fine tuning}

Before we exploit fully the two constraints $\mu^{2} \geq \mu_{l b}^{2}, M_{A} \geq\left(M_{A}\right)_{l b}$ to restrict the parameter space, we apply the general criterion that the expression (10) is satisfied without excessive tuning among the various terms $[22,10]$. In radiative EWSB the physical value of $M_{Z}$, which sets the EW scale, may be written as

$$
M_{Z}^{2}=2\left(J_{1} m_{1 / 2}^{2}+J_{2} m_{0}^{2}+J_{3} A_{0}^{2} m_{0}^{2}+J_{4} A_{0} m_{0} m_{1 / 2}-\mu^{2}\right) .
$$

Accidental compensation (fine tuning) among different terms in Eq. (12) may occur. We explicitly require the absence of too-strong fine tuning, i.e., carcellations among exceedingly large values of the parameters $m_{1 / 2}$, $m_{0}, A_{0}$ and $\mu$. Denoting by $\eta_{f}$ a parameter which quantifies the degree of fine tuning, we require [22] that

$$
\left|\frac{\Delta M_{Z}^{2}}{M_{Z}^{2}}\right|<\eta_{f}\left|\frac{\Delta x_{i}^{2}}{x_{i}^{2}}\right|
$$

where $x_{i}$ denotes any of the previous parameters. For instance, for $A_{0}=0$, Eq. (13) provides the following conditions

$$
m_{1 / 2}^{2}<\frac{\eta_{f}}{2\left|J_{1}\right|} M_{Z}^{2}, \quad m_{0}^{2}<\frac{\eta_{f}}{2\left|J_{2}\right|} M_{Z}^{2}, \quad \mu^{2}<\frac{\eta_{f}}{2} M_{Z}^{2} \simeq(640 \mathrm{GeV})^{2}
$$


where in the last approximate equality we have taken $\eta_{f}=100$, which means that we allow an accidental compensation at the $1 \%$ level. The upper bound on $m_{0}$ depends on $\tan \beta$ and the $\delta_{i}$ 's, whereas that on $m_{1 / 2}$ varies only with $\tan \beta$ (because of the nature of the Ansatz (8): see the comment after Eq. (8)).

For the sake of illustration, we give some numerical examples, taking again $\eta_{f}=100$. For $\tan \beta=8$, we have, for $\delta_{1}=\delta_{2}=0, m_{1 / 2} \lesssim 400 \mathrm{GeV}, m_{0} \lesssim 1.5 \mathrm{TeV}$. For two other pairs of values of the $\delta_{i}$ 's, which will be discussed later on, we obtain $m_{0} \lesssim 2.4 \mathrm{TeV}$ for $\delta_{1}=-0.2, \delta_{2}=0.4$ and $m_{0} \lesssim 3.0 \mathrm{TeV}$ for $\delta_{1}=-0.8, \delta_{2}=0.2$. At $\tan \beta=53$ we have $m_{1 / 2} \lesssim 415 \mathrm{GeV}$ and $m_{0} \lesssim(1.7-1,9) \mathrm{TeV}$, depending on the values for the $\delta_{i}$ 's. These inequalities imply for the neutralino mass $m_{\chi} \lesssim 170 \mathrm{GeV}$.

In the following, when graphical representations for the parameter space are shown, we display no-fine-tuning upper bounds obtained from the general expression (13) with $\eta_{f}=100$. These upper bounds are denoted by dashed lines in Figs. 9-14.

\section{Constraints due to radiative EWSB}

The EWSB constraints are given by the set of Eqs. (5)-(7), or equivalently by Eqs. (10)-(11), together with the conditions $\mu^{2} \geq \mu_{l b}^{2}$ and $M_{A} \geq\left(M_{A}\right)_{l b}$. From these equations the values of $m_{0}$ and $m_{1 / 2}$ (or $\mu$ and $\left.m_{1 / 2}\right)$ are constrained and thus some domains in the $\left(m_{1 / 2}, m_{0}\right)$ or $\left(m_{1 / 2}, \mu\right)$ planes can be excluded. Let us start this discussion by analyzing the condition $M_{A} \geq\left(M_{A}\right)_{b}$, with $M_{A}$ given by Eq. (11). For the sake of simplicity, we put $A_{0}=0$ for the moment. To discuss the role of $M_{A} \geq\left(M_{A}\right)_{l b}$ in placing bounds on $m_{1 / 2}$ and $m_{0}$, we first rewrite it explicitly as

$$
K_{1} m_{1 / 2}^{2}+K_{7} m_{0}^{2} \geq M_{Z}^{2}+\left(M_{A}\right)_{l b}^{2} .
$$

The nature of this quadratic form in the $\left(m_{1 / 2}, m_{0}\right)$ plane obviously depends on the signs of the two coefficients $K_{1}$ and $K_{2}$. As we have seen in Sect.II, it turns out that, whereas $K_{1}$ is always positive, the sign of $K_{2}$ depends on the values of $\tan \beta$ and of the $\delta_{i}$ 's. Two different situations may occur, depending on the sign of $K_{2}$. In the case $K_{2}>0$ the region allowed by (15) is the one above an elliptical branch centered in the origin of the $\left(m_{1 / 2}, m_{0}\right)$ plane. Therefore, both parameters $m_{1 / 2}$ and $m_{0}$ are bounded from below. When $K_{2}<0$, the region allowed by Eq. (15) is the one between the $m_{1 / 2}$ axis and an upward-moving hyperbolic branch. Thus, whereas $m_{1 / 2}$ is still bounded from below, $m_{0}$ is now constrained from above. The upper bound on $m_{0}$ is particularly stringent when $K_{2}$ is large and negative and $K_{1}$ is not large. This occurs, for instance, at very large values of $\tan \beta$ in the case of $m_{0}$ universality.

This discussion may be extended straightforwardly to the case $A_{0} \neq 0$. In this case the constraint $M_{A} \geq$ $\left(M_{A}\right)_{l b}$ may be written explicitly as

$$
K_{1} m_{1 / 2}^{2}+K_{2} m_{0}^{2}+K_{3} A_{0}^{2} m_{0}^{2}+K_{4} A_{0} m_{0} m_{1 / 2} \geq M_{Z}^{2}+\left(M_{A}\right)_{l b}^{2} .
$$

The nature of this quadratic form depends on the sign of its determinant. When this determinant is positive, an elliptical branch in the $\left(m_{1 / 2}, m_{0}\right)$ plane provides lower bounds on the two variables. On the other hand, a negative determinant entails an upward-moving hyperbolic branch which places an upper bound on $m_{0}$. These branches are part of conics whose axes are somewhat tilted with respect to the $\left(m_{1 / 2}, m_{0}\right)$ axes.

Similar implications follow from the constraint $\mu^{2} \geq \mu_{l b}^{2}$, which may be written explicitly as (for $A_{0}=0$ )

$$
J_{1} m_{1 / 2}^{2}+J_{2} m_{0}^{2} \geq \frac{M_{Z}^{2}}{2}+\mu_{l b}^{2}
$$

This quadratic form may be discussed in much the same way as the one in Eq. (15). From the properties seen in Section 2 it turns out that the coefficient $J_{1}$ is always positive, whereas the coefficient $J_{2}$ is positive in the universal case, but may be negative when deviations from $m_{0}$ universality are introduced. Thus it follows 
that the condition $\mu^{2} \geq \mu_{l b}^{2}$ puts lower bounds on $m_{1 / 2}$ and either lower or upper bounds on $m_{0}$, depending on the sign of $J_{2}$ (due to analytic properties identical to those discussed previously below Eq. (15)). The condition $\mu^{2} \geq \mu_{l b}^{2}$ sets a very stringent upper bound on $m_{0}$, whenever $J_{2}$ is negative and large in magnitude. The extension to the case $A_{0} \neq 0$ may be repeated here in a way similar to the above discussion for Eq. (16).

Thus we have seen that two important constraints, $\mu^{2} \geq \mu_{l b}^{2}$ and $M_{A} \geq\left(M_{A}\right)_{l b}$, are at work in bounding $m_{1 / 2}$ and $m_{0}$, when EWSB is required to occur radiatively. When $J_{2}$ and $K_{2}$ are positive, the two conditions place lower bounds on $m_{1 / 2}$ and $m_{0}$. Similar constraints are established by the requirements that also the sfermion masses and $m_{X}$ satisfy the relevant experimental bounds. These last conditions are not explicitly discussed here, but they are taken into account in our evaluations.

It is worth emphasizing that the most dramatic impact of the conditions $\mu^{2} \geq \mu_{l b}^{2}$ and $M_{A} \geq\left(M_{A}\right)_{l b}$ over the parameter space occurs when either $J_{2}$ or $K_{2}$ (or both of them) are negative. Under these circumstances, as we have seen above, $\mu^{2} \geq \mu_{l b}^{2}$ and $M_{A} \geq\left(M_{A}\right)_{l b}$ may place stringent upper limits on $m_{0}$, bounding the neutralino parameter space considerably. Which of the two conditions prevails over the other depends on the specific regions of the full parameter space and on the values of the $\delta_{i}$ 's. In Section 7 we will illustrate the implications of these constraints in a few specific examples.

\section{Cosmological constraint}

Let us turn now to the evaluation of the neutralino relic abundance $\Omega_{X} h^{2}$ and to the requirement that the lightest neutralino is not overproduced, i.e., $\Omega_{\chi} h^{2} \leq 1$.

The neutralino relic abundance $\Omega_{\chi} h^{2}$ is evaluated following the standard procedure [23-26], according to which $\Omega_{\chi} h^{2}$ is essentially given by $\Omega_{\chi} h^{2} \propto\left\langle\sigma_{\text {ann }} v\right\rangle_{\text {int }}^{-1}$, where $\left\langle\sigma_{\text {ann }} v\right\rangle_{\text {int }}$ is the thermally-averaged annihilation cross section, integrated from the freeze-out temperature to the present temperature. The standard expansion $\left\langle\sigma_{\mathrm{ann}} v\right\rangle=a+b x+\cdots$ may be employed, with $x=T / m_{\chi}$, except at s-channcl resonances $(Z, A, H, h)$, where a more precise treatment has to be used for the thermal average [24]. In the evaluation of $\left\langle\sigma_{\text {ann }} v\right\rangle$ the full set of annihilation final states ( $f \bar{f}$ pairs, gauge-boson pairs, Higgs-boson pairs and Higgs-gauge boson pairs), as well as the complete set of Born diagrams are taken into account [26]. We recall that one of the largest contributions to the annihilation cross section is provided by diagrams with the exchange of the pseudoscalar Higgs boson $A$. (More relevant properties of the Higgs bosons are discussed in Section 8.) We note that the constraint $\Omega_{X} h^{2} \leq 1$ is very effective for small and intermediate values of $\tan \beta$, but is not restrictive for large values of $\tan \beta$. The strong restriction in the former case comes from the large value of $M_{A}$ implied by small and intermediate values of $\tan \beta$ (see Fig. 5) (also the couplings of $A$ to $\chi$ and fermions are small for these values of $\tan \beta$ ).

We show in Figs. 6-8 a few examples where $\Omega_{\chi} h^{2}$ is given as a function of $m_{\chi}$ in the form of scatter plots. These scatter plots have been obtained by varying the parameters $m_{0}$ and $m_{1 / 2}$ on a equally-spaced linear grid over the ranges $10 \mathrm{GeV} \leq m_{0} \leq 2 \mathrm{TeV}, 45 \mathrm{GeV} \leq m_{1 / 2} \leq 500 \mathrm{GeV}$. Furthermore, we remark that all evaluations presented in this paper are for positive values of $\mu$, since negative values of $\mu$ are disfavoured by the constraints due to $m_{b}$ and $b \rightarrow s \gamma$ processes (see Section 6 ). The configurations shown in Figs. 6-8 satisfy the constraints due to radiative EWSB, discussed previously.

In Fig. 6 is shown the case $\tan \beta=8$ and $\delta_{i}=0$. Here, as expected because of the intermediate value of $\tan \beta$, many neutralino configurations provide $\Omega_{\chi} h^{2}>1$, whilst only a few give $\Omega_{\chi} h^{2} \leq 1$. (Also, $M_{A}$ is large here because of sizeable values of $K_{2}$ (see Fig. 2d), which helps increase $\Omega_{\chi} h^{2}$.) An exception occurs when $m_{\chi} \simeq M_{Z} / 2$, since in this case the annihilation cross section is greatly enhanced due to the Z-pole contribution.

In Fig. 7 we display $\Omega_{X} h^{2}$ in a case of non-universality ( $\delta_{1}=-0.2, \delta_{2}=0.4$, for definiteness). It is easier to find $\Omega_{\chi} h^{2} \leq 1$ in this case, since here the departure from $m_{0}$ universality implies a changeover of the neutralino composition from the gaugino dominance of the previous example to higgsino dominance (this point will be elucidated in Section 7). This implies a larger $\chi-\chi$ annihilation cross section and consequently a smaller relic 


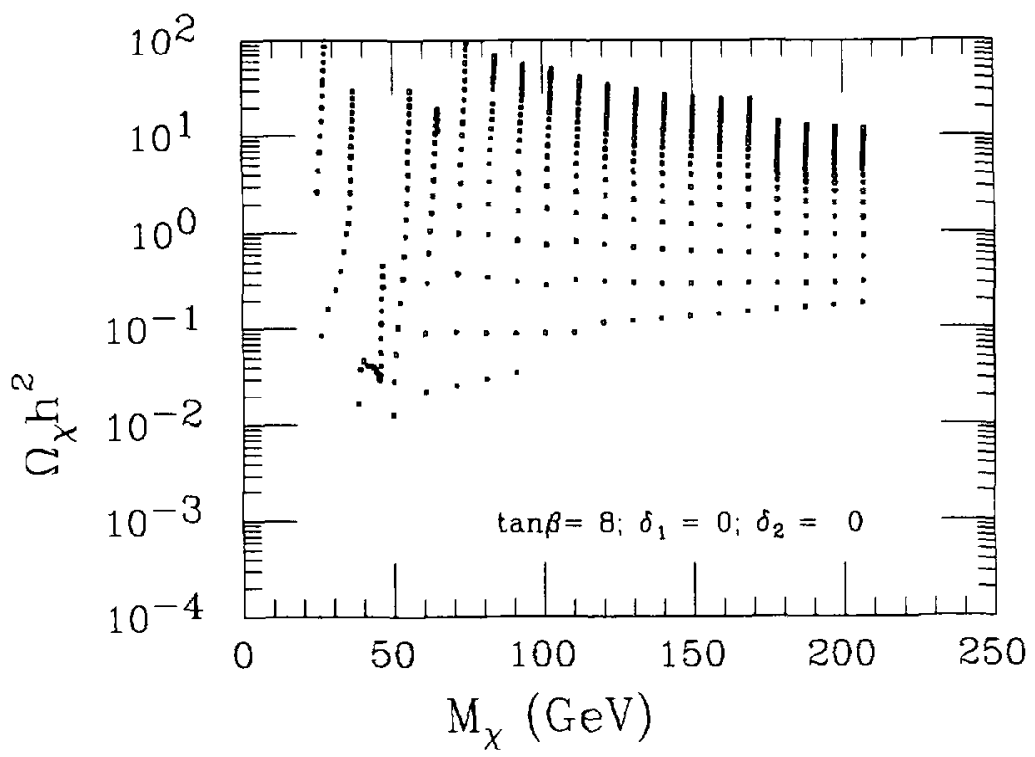

Fig. 6. Scatter plot representing $\Omega_{X} h^{2}$ as a function of $m_{X}$ for $\tan \beta=8, \delta_{1}=0$ and $\delta_{2}=0$. Parameters are varied on a linear equally-spaced grid over the ranges: $10 \mathrm{GeV} \leq m_{0} \leq 2 \mathrm{TeV}, 45 \mathrm{GeV} \leq m_{1 / 2} \leq 500 \mathrm{GeV}$.

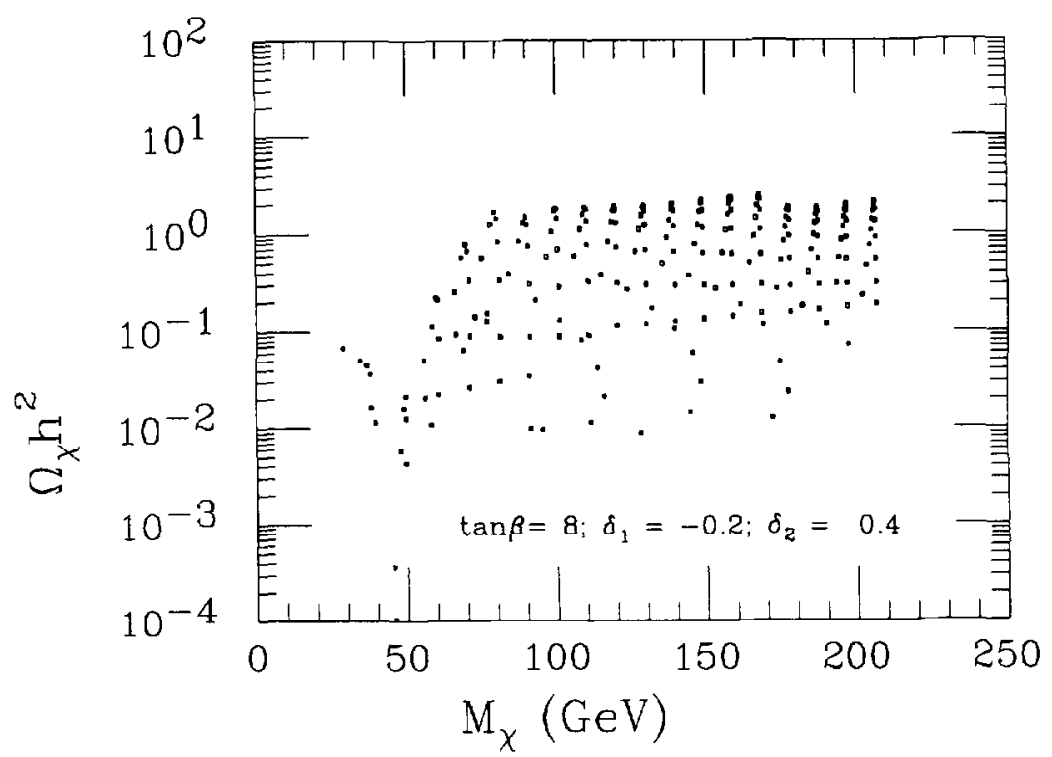

Fig. 7. The same as in Fig. 6 , but with $\delta_{1}=-0.2$ and $\delta_{2}=0.4$.

abundance. Thus only a few neutralino configurations are excluded by the $\Omega_{X} h^{2} \leq 1$ condition.

An example for $\Omega_{\chi} h^{2}$ in the case of large $\tan \beta$ and $\delta_{i}=0$ is shown in Fig. 8. We see that $\Omega_{\chi} h^{2} \leq 1$ imposes no constraint since, for this very large value of $\tan \beta$, annihilation cross sections are very large. 


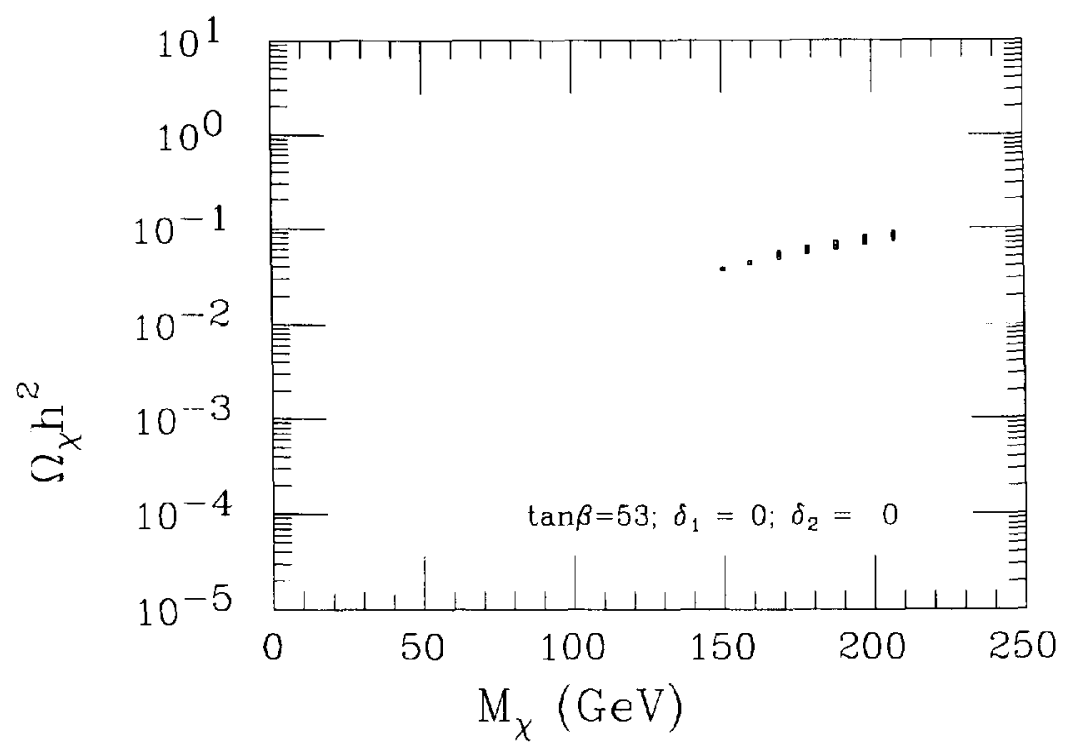

Fig. 8. Scatter plot representing $\Omega_{\chi} h^{2}$ as a function of $m_{\chi}$ for $\tan \beta=53, \delta_{1}=0$ and $\delta_{2}=0$. Parameters ranges are as in Fig. 6 .

\section{Constraints from $b \rightarrow s \gamma$ and $m_{b}$}

In the evaluation of the $b \rightarrow s \gamma$ decay rate we have included the supersymmetric contributions arising from the charged Higgs loops and chargino loops given in Ref. [27]. The Higgs term always adds to the Standard Model value and usually entails too large a value for the rate. On the other hand, the chargino contribution gives rise to a destructive interference for $\mu>0$ (in our convention for the sign of $\mu$ ). At large $\tan \beta$ supersymmetric contributions may be sizeable: unless the destructive interference protects the decay rate, it can very easily be driven out of the present experimental bounds. In the light of this property, the positive $\mu$ scenario appears to be the favourite one and, as already remarked, in this paper we only show results for this case. In comparing our predictions with observations we have taken into account that, as discussed in Ref. [28], large theoretical uncertainties are present, mainly due to QCD effects. In particular, predictions depend very strongly on the choice of the renormalization scale, leading to an inaccuracy of order $25 \%$. To account for this effect we have relaxed the experimental bounds of Ref.[29] by the same amount, keeping the renormalization scale fixed at the representative value of $5 \mathrm{GeV}$. Thus, our requirement is that the rate of $b \rightarrow s \gamma$ decay falls into the range $0.8 \times 10^{-4} \leq \mathrm{BR}(b \rightarrow s \gamma) \leq 5.3 \times 10^{-4}$.

The supersymmetric corrections to the bottom mass include contributions from bottom-squark-gluino loops and from top-squark-chargino loops [30]. In the present analysis, the bottom mass is computed as a function of the other parameters and required to be compatible with the present experimental bounds. Theoretical uncertainties in the evaluations of $m_{b}$ arise both from the running of the RGE's and from assumptions about Yukawa unification. Since our choice is to solve RGE's at the 1-loop level and without thresholds, we estimate an uncertainty of the order of $10 \%$ in our prediction for $m_{b}$. In addition, a relatively small departure (see Ref. [31 ]) from bottom- $\tau$ Yukawa unification at the GUT scale may significantly change the bottom mass result. To take into account such uncertainties we have chosen to weaken the bounds on $m_{l}$, given in [32] by an amount of $10 \%$. Thus we require $m_{b}$ to fall into the range $2.4 \mathrm{GeV} \leq m_{b}\left(M_{Z}\right) \leq 3.7 \mathrm{GeV}$. 


\section{Allowed regions in neutralino parameter space}

We discuss now in a few examples how the various constraints analysed in the previous sections complement each other in shaping the allowed regions in the parameter space. We start with the $\left(m_{1 / 2}, m_{0}\right)$ representation, and later display our results in the $\left(m_{1 / 2}, \mu\right)$ plane which provides the most useful representation for neutralino phenomenology.

Let us first clarify a few graphical conventions adopted in our $\left(m_{1 / 2}, m_{0}\right)$ and $\left(m_{1 / 2}, \mu\right)$ plots. Regions are left empty when at least one of the following constraints is not satisfied: (i) experimental bounds on Higgs, neutralino and sfermion masses [16,33], (ii) the $\chi$ is the Lightest Supersymmetric Particle (LSP), (iii) radiative EWSB and $\mu^{2} \geq \mu_{l b}^{2}, M_{A} \geq\left(M_{A}\right)_{l b}$. Regions forbidden by the cosmological constraint $\left(\Omega_{\chi} h^{2} \leq 1\right)$ are explicitly denoted by dots and those disallowed by the $b \rightarrow s \gamma, m_{b}$ constraints (but not by the previous ones) are denoted by crosses (crosses are displayed only in the $\left(m_{1 / 2}, m_{0}\right)$ plane, but not in the $\left(m_{1 / 2}, \mu\right)$ plane, to simplify these plots). The allowed domains are denoted by squares when they satisfy $\Omega_{\chi} h^{2}>0.01$, or by diamonds otherwise in the $\left(m_{1 / 2}, m_{0}\right)$ plots. They are denoted by squares in the $\left(m_{1 / 2}, \mu\right)$ plots, independently of the $\Omega_{X} h^{2}$ value. To simplify the discussion, we first take $A_{0}=0$. We comment on the $A_{0} \neq 0$ case at the end of this section.

As a first example, let us consider the representative point $\tan \beta=8$. For this intermediate value of $\tan \beta$, the cosmological constraint is expected to be very effective in view of the arguments discussed in Section 5 . This is actually the case for universal $m_{0}$, when both $K_{2}$ and $J_{2}$ are positive (see Fig. 2), so that the conditions of radiative EWSB do not set any upper limit on $m_{0}$ (Fig. 9a). The empty region in the lower part of these figures is forbidden by the experimental bound on $m_{\chi}$. As shown in this figure, in wide regions (denoted by dots) $\Omega_{\chi} h^{2}>1$. Thus the cosmological constraint places a very stringent upper bound on $m_{0}$ for $m_{1 / 2} \gtrsim 150$ $\mathrm{GeV}$. However, for smaller values of $m_{1 / 2}$, an allowed horizontal region extends up to $m_{0} \simeq 2 \mathrm{TeV}$. In fact, along this strip, $m_{\chi} \simeq M_{Z} / 2$ and then $\Omega_{\chi} h^{2} \leq 1$ is satisfied (see the discussion in Section 5).

Moving away from the universal point towards a region where $J_{2}$ is negative, we expect $\mu^{2} \geq \mu_{l b}^{2}$ to bc effective in placing a stringent upper bound on $m_{0}$. This is actually the case in the example shown in Fig. 10a, which refers to the representative point $\delta_{1}=-0.2, \delta_{2}=0.4\left(J_{2}=-0.07\right)$. Here it is the bound $\mu^{2} \geq \mu_{l b}^{2}$ which provides the most stringent constraint in disallowing the large (empty) domain on the right side. Nevertheless, $\Omega_{\chi} h^{2} \leq 1$ is still effective in excluding an internal region that would otherwise be allowed (see the discussion below).

Keeping $\tan \beta=8$, we complete our discussion by considering the representative point $\delta_{1}=-0.8, \delta_{2}=0.2$ shown in Fig. $11 \mathrm{a}$, which gives an example where $J_{2}$ is very small. The peculiarity of this example will become clear when we discuss the relevant situation in the $\left(m_{1 / 2}, \mu\right)$ plane, to which we now turn.

The shape and general properties of the physical region in the $\left(m_{1 / 2}, \mu\right)$ plane are dictated by the constraints previously derived, and they are determined most notably by $J_{2}$. It is convenient to distinguish the two cases (i) $J_{2}>0$ and (ii) $J_{2}<0$. For case (i) at fixed $m_{1 / 2}, \mu$ increases for increasing $m_{0}$ with the consequence that the allowed physical region extends to the right of the $m_{0}=m_{0, \min }$ line in the $\left(m_{1 / 2}, \mu\right)$ plane, allowing for the neutralino only a gaugino-dominated region $\left(m_{0, \min }\right.$ is the minimum allowed value of $\left.m_{0}\right)$. In the case (ii) $\left(J_{2}<0\right)$, starting from the $m_{0}=m_{0, n i n}$ line and increasing $m_{0}$ at fixed $m_{1 / 2}$, one moves to the left and then one may reach regions of sizeable higgsino-gaugino mixing or even of higgsino dominance. Case (i) applies in particular to the case of $m_{0}$ universality $\left(\delta_{i}=0\right)$ for any value of $\tan \beta$. This is clear from Fig. 3, which shows that in the $\left(\delta_{2}, \delta_{1}\right)$ plane the origin is below any $J_{2}=0$ line. An example of this situation is displayed in Fig. $9 \mathrm{~b}$ ( for $\tan \beta=8$ ).

However, as we have seen in Section 2, when the assumption of $m_{0}$ universality is relaxed, then $J_{2}$, which in the universal case is positive and small, may very easily become negative and sizeable. In this case a changeover in neutralino composition from an originally gaugino-like state into a higgsino-like one occurs. This remarkable property, discussed in Ref. [14] for large $\tan \beta$, is in fact valid over the whole range of $\tan \beta$, if the degree of non-universality is increased for decreasing $\tan \beta$. An example of case (ii) $\left(J_{2}<0\right)$ is shown in Fig. 10b, 
(a)

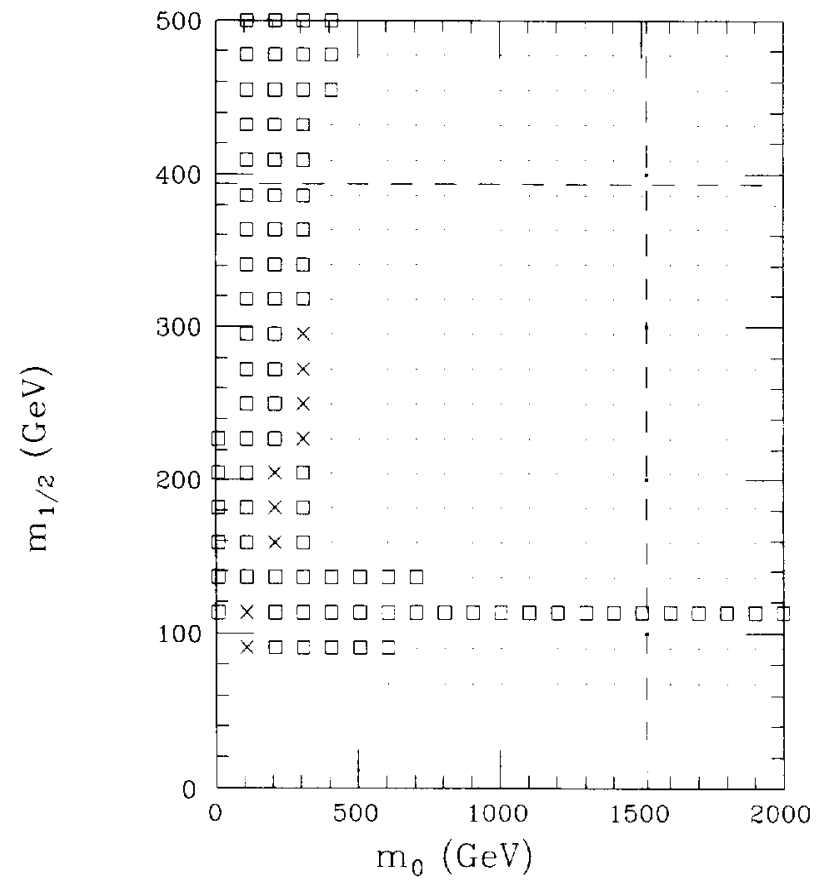

(b)

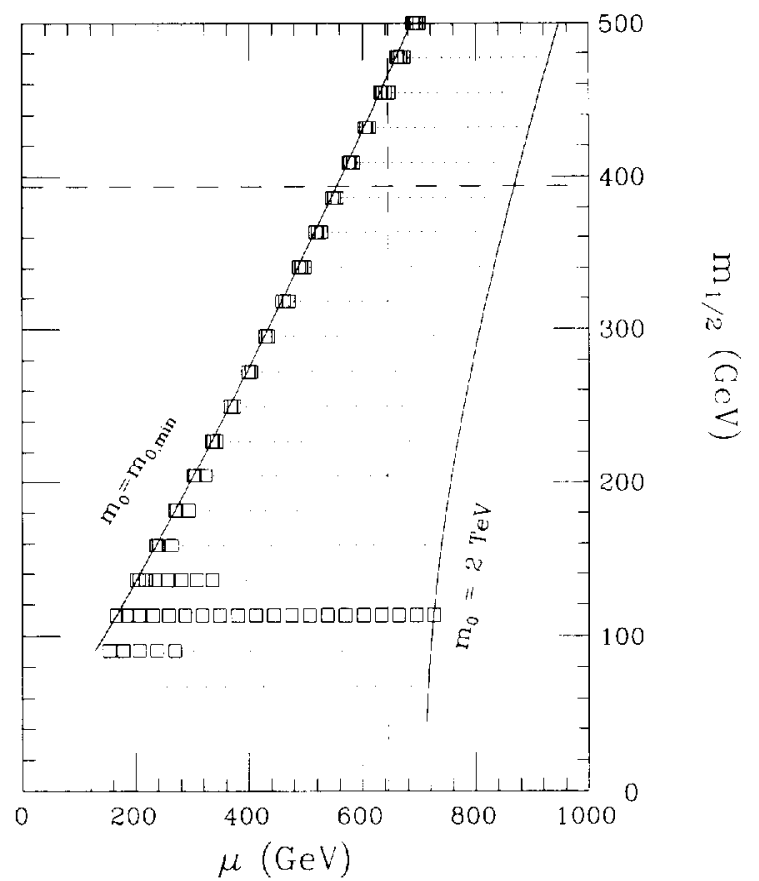

Fig. 9. (a) Parameter space in $\left(m_{1 / 2}, m_{0}\right)$ plane for $\tan \beta=8, \delta_{1}=0$ and $\delta_{2}=0$. Empty regions are excluded by: (i) accelerator constraints, (ii) radiative EWSB conditions, (iii) neutralino is not the LSP. Dots represent the region where $\Omega_{\chi} h^{2}>1$. Regions with crosses are excluded by $b \rightarrow s \gamma$ and $m_{b}$ constraints. In the regions denoted by squares, $0.01<\Omega_{\chi} h^{2} \leq 1$. The region without fine-tuning is inside the box bounded by dashed lines. (b) Parameter space represented in the $\left(m_{1 / 2}, \mu\right)$ plane. Solid lines correspond to the extreme values of $m_{11}$. Notations are the same as in (a), but crosses are omitted here.

where the allowed region extends widely into the higgsino region. It is instructive to compare Fig. 9 with Fig. 10. Looking at sections (a) of these figures, we notice that changing the values of the $\delta_{i}$ 's from the set $\delta_{i}=0$ to the set $\delta_{1}=-0.2, \delta_{2}=0.4$ relaxes substantially the cosmological constraint. Parts (b) of these figures provide the explanations for this feature. In fact, whereas in the former case the neutralino is mainly a gaugino, in the latter case $\chi$ is higgsino-like or mixed. As we already remarked, this implies an increase of the $\chi-\chi$ annihilation cross section and a reduction of the relic abundance. The physical region also displays an extension to the right, in the example of Fig. $11 \mathrm{~b}$, but here the effect is very tiny, due to a very small $J_{2}$ and to the severe upper bound on $m_{0}$ for $m_{1 / 2} \gtrsim 180 \mathrm{GeV}$. This is the first case to show a very marked $\left(m_{1 / 2}, \mu\right)$ correlation.

Now we turn to the case of large $\tan \beta$, where new features appear. First, the $M_{A} \geq\left(M_{A}\right)_{l b}$ condition is no longer protected by large values of $K_{1}$, and may become effective in restricting the parameter space. Secondly, the $m_{b}$ and $b \rightarrow s \gamma$ conditions are now rather stringent over large domains and not only occasionally relevant as in the smaller $\tan \beta$ cases. Thirdly, the cosmological constraint is usually overwhelmed by the other conditions. In Figs. 12a, 13a, 14a we have, for $\tan \beta=53$, the following sequence of examples. (i) $\delta_{1}=0, \delta_{2}=0$ (Fig. 12a): here $K_{2}<0, J_{2}>0$, and since $K_{2}$ is negative and sizeable in magnitude, the constraint $M_{A} \geq\left(M_{A}\right)_{l b}$ sets an extremely stringent upper bound on $m_{0}$ and thus forbids the wide (empty) region on the right. (ii) $\delta_{1}=0, \delta_{2}=-0.3$ (Fig. 13a): here one still has $K_{2}<0, J_{2}>0$, but $\left|K_{2}\right|$ is smaller than in the previous case, so the constraint $M_{A} \geq\left(M_{A}\right)_{b}$ is still very effective but less compelling than in the case (i). Also, the role of the $m_{b}$ and the $b \rightarrow s \gamma$ conditions is more significant here. (iii) $\delta_{1}=0.7, \delta_{2}=0.4$ (Fig. 14a): here $K_{2}>0$, 
(a)

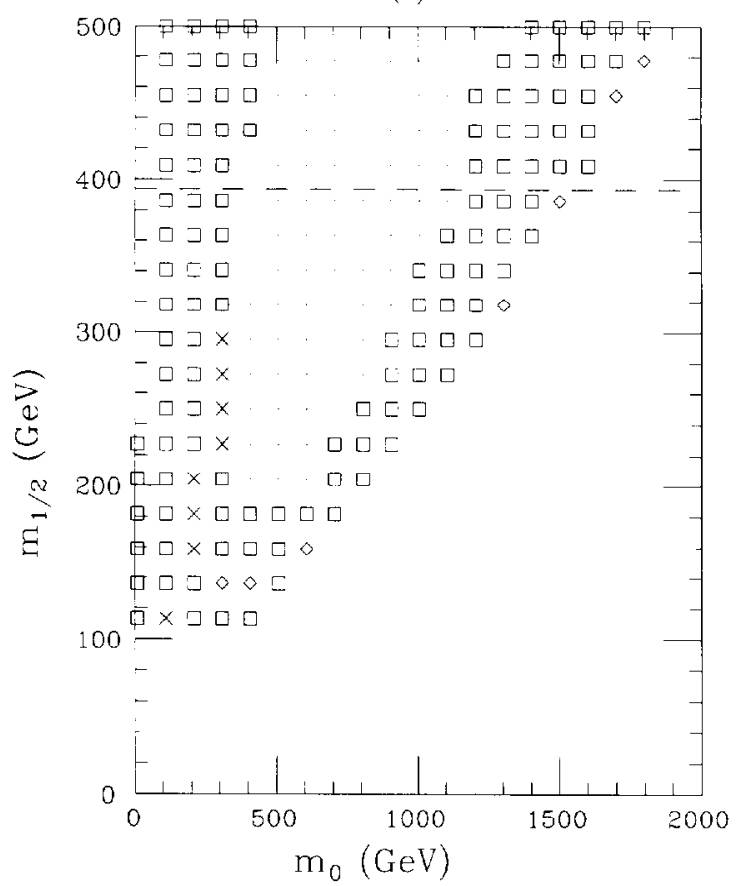

(b)

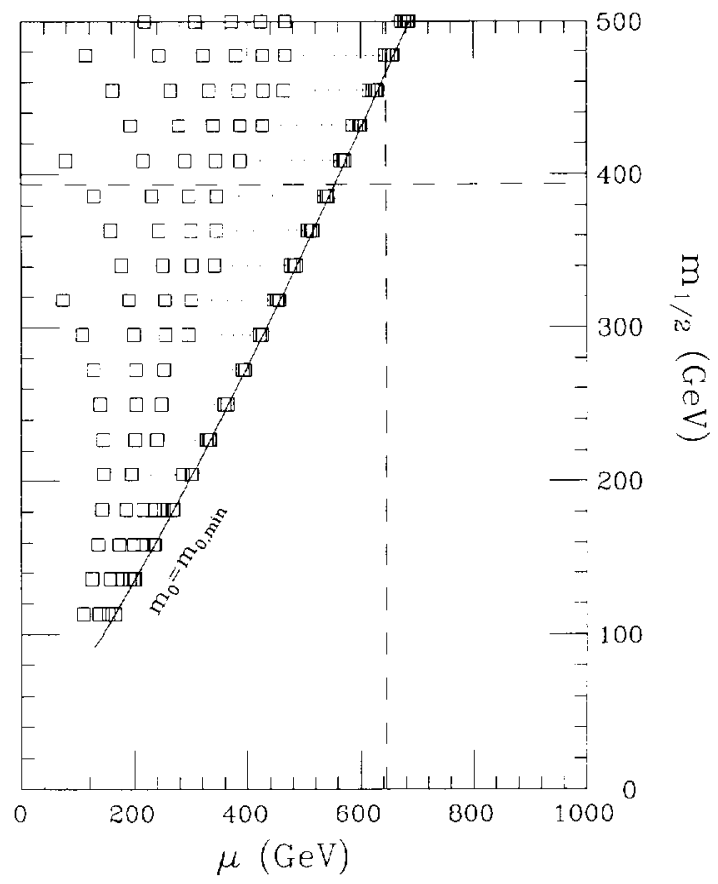

Fig. 10. (a) The same as in Fig. 9, but with $\delta_{1}=-0.2$ and $\delta_{2}=0.4$. In the regions denoted by diamonds $\Omega_{\chi} h^{2} \leq 0.01$. (b) Notations are the same as in (a). Note that the domain where the neutralino is the dark matter particle with $0.01<\Omega_{\chi} h^{2} \leq 1$ has shifted to the higgsino-dominated region. Crosses are omitted here.

$J_{2}<0, M_{A} \geq\left(M_{A}\right)_{l b}$ gives a lower bound on $m_{1 / 2}$ and the $\mu^{2} \geq \mu_{l h}^{2}$ condition provides the frontier of the empty domain on the right.

The $\left(m_{1 / 2}, \mu\right)$ representations for large $\tan \beta$ and for the representative $\delta_{i}$ points discussed above are displayed in Figs. 12b-14b. We start from the universal case of Fig. 12b. Here we expect gaugino-dominated configurations. However, hecause the values of $m_{0}$ are strongly limited from above (see Fig. 12a), we have the extremely correlated states shown in Fig. 12b. In the case of Fig. 13b one has $J_{2}>0$, and gaugino-dominated states occur. No strong $\left(m_{1 / 2}, \mu\right)$ correlation shows up in this case. The opposite case, $J_{2}<0$, is shown in Fig. 14b, where higgsino-dominated configurations appear.

It is worth adding a few comments about the examples of Figs. 11 and 12, where the physical regions in the $\left(m_{1 / 2}, \mu\right)$ plane show a very pronounced correlation in the two variables. This feature occurs whenever $\left|J_{2}\right| m_{0}^{2} \ll J_{1} m_{1 / 2}^{2}$, i.e., whenever $m_{0}$ is severely bounded from above and/or $\left|J_{2}\right|$ is very close to zero. As far as the values of $\left|J_{2}\right| / J_{1}$ are concerned, we notice that in the universal case (see Fig. 2c), except for small values of $\tan \beta, J_{2} / J_{1} \simeq 0.04$ (in fact, for $\tan \beta \gtrsim 4, J_{1} \simeq-a_{2} \simeq 2.5, J_{2} \simeq-b_{2} \simeq 0.1$ ). Thus for $\delta_{i}=0 \mathrm{a}$ strong $\left(m_{1 / 2}, \mu\right)$ correlation occurs whenever $m_{0} \lesssim O\left(m_{1 / 2}\right)$. This happens in the example of Fig. 12, where $m_{0}$ is severely bounded by the $M_{A} \geq\left(M_{A}\right)_{l b}$ condition, and in the case of Fig. 11, where the correlation is enforced by a very small value of $J_{2}: J_{2}=0.06$. A $\left(m_{1 / 2}, \mu\right)$ correlation is also exhibited in Fig. $9 \mathrm{~b}$ for the range $m_{1 / 2} \gtrsim 150 \mathrm{GeV}$, where $m_{0}$ is bounded by the cosmological constraint.

In general, we do not consider these physical regions with a strong $\left(m_{1 / 2}, \mu\right)$ correlation as unnatural, since they are usually realized without much tuning. We recall that the size of the coefficients $J_{1}$ and $J_{2}$ is dictated by the RGEs with their intrinsic cancellations, and that one naturally has $J_{1}=O(\mathrm{a}$ few $), J_{2}=O(0.1-0.01)$. As we have seen, these properties, combined with severe upper bounds on $m_{0}$, are sufficient to generate the $\left(m_{1 / 2}\right.$, $\mu)$ correlation. 
(a)

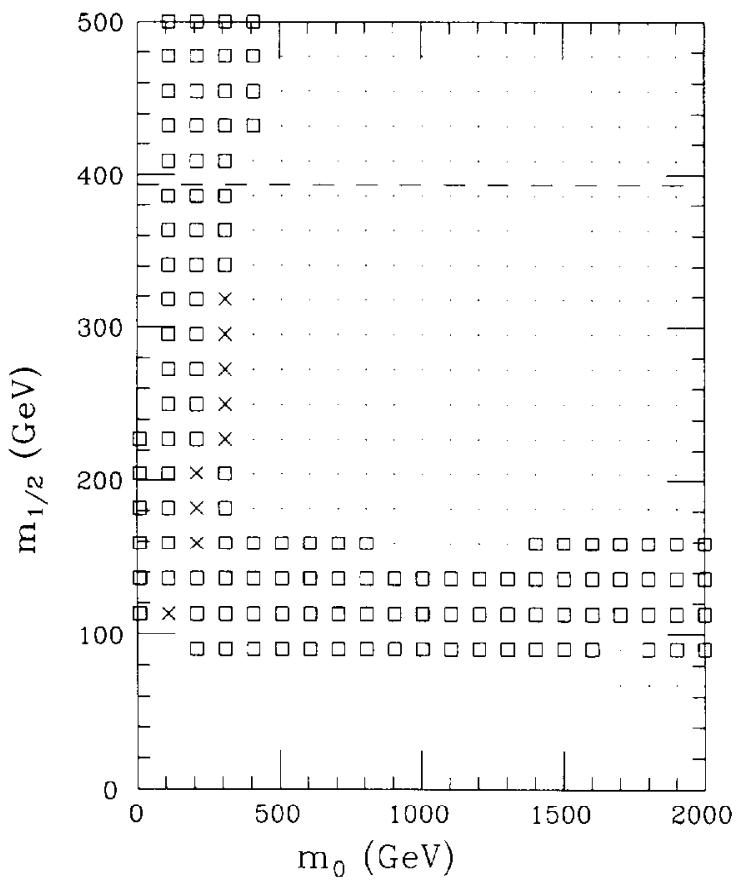

(b)

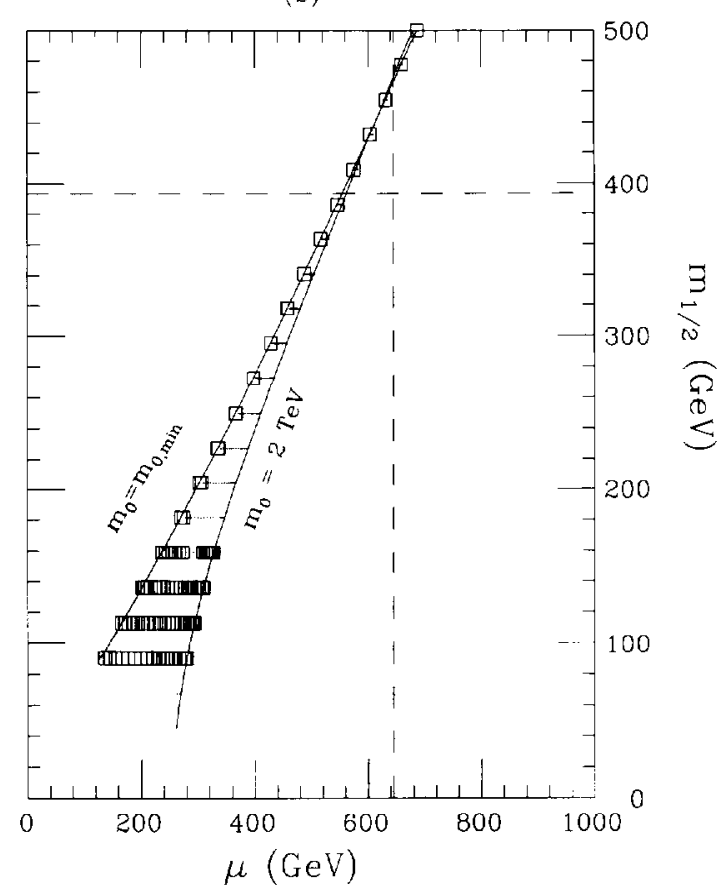

Fig. 11. The same as in Fig. 9 , but with $\delta_{1}=-0.8$ and $\delta_{2}=0.2$.

We turn now to the $A_{0} \neq 0$ case. First we recall that $A_{0}$ is constrained in the range $\left|A_{0}\right| \lesssim 3$ from the absence of charge and color breaking [34]. Thus, allowing $A_{0} \neq 0$ does not change essentially the general picture previously discussed. The previous scenarios still occur, but at different points in the parameter space. Two specific comments are in order here: (i) independently of its sign, $A_{0}$ disfavours the changeover from gaugino dominance to higgsino dominance in the neutralino composition, (ii) a negative $A_{0}$ reduces the value of $M_{A}$ as compared to the $A_{0}=0$ case, and so either provides a light $A$ boson (and hence interesting phenomenology) or enforces a more stringent constraint on the parameter space.

\section{Neutral Higgs bosons}

Neutralino direct detection, to be discussed in the next section, is based on neutralino-nucleus scattering. In this process, exchanges of neutral Higgs bosons play a dominant role, provided the Higgs masses are not too heavy. It is convenient to recall here some relevant properties of the couplings of $\chi$ with matter via Higgs exchange. As was already mentioned in the Introduction, the two Higgs isodoublets $H_{1}, H_{2}$ yicld 3 ncutral Higgs mass eigenstates: one CP-odd $(A)$ state, whose mass $M_{A}$ is given by expression (11) and two CP-even states (of masses $M_{h}, M_{H}, M_{h}<M_{H}$ ), which are obtained from $H_{\mathrm{l}}^{0}, H_{2}^{0}$ by a rotation through an angle $\alpha$

$$
H=\cos \alpha H_{1}^{0}+\sin \alpha H_{2}^{0}, \quad h=-\sin \alpha H_{1}^{0}+\cos \alpha H_{2}^{0} .
$$

It is important to notice here that $\alpha$ depends very sensitively on $M_{A}$, being very close to zero for $\tan \beta \gtrsim 4$ and rising very fast to $\pi / 2$ for $M_{A} \lesssim O\left(M_{Z}\right)$ (see Fig. 15).

The angle $\alpha$ plays a crucial role in determining the size of the neutral $h, H$-quark couplings. Here, as we are interested in $\chi$-nucleus scattering, we discuss explicitly only the couplings involving the CP-even states, since 
(a)

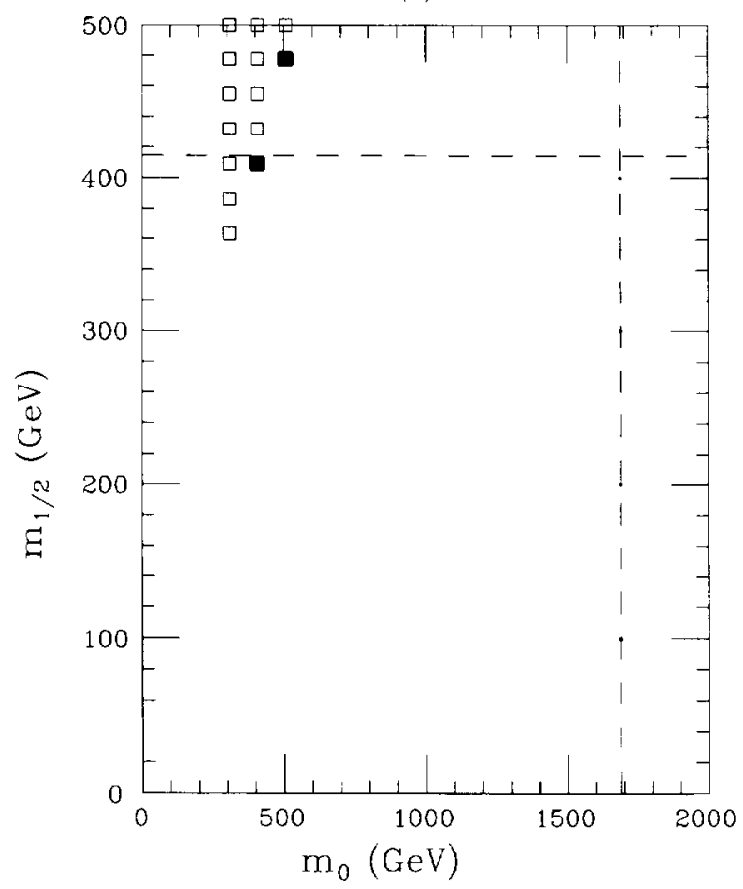

(b)

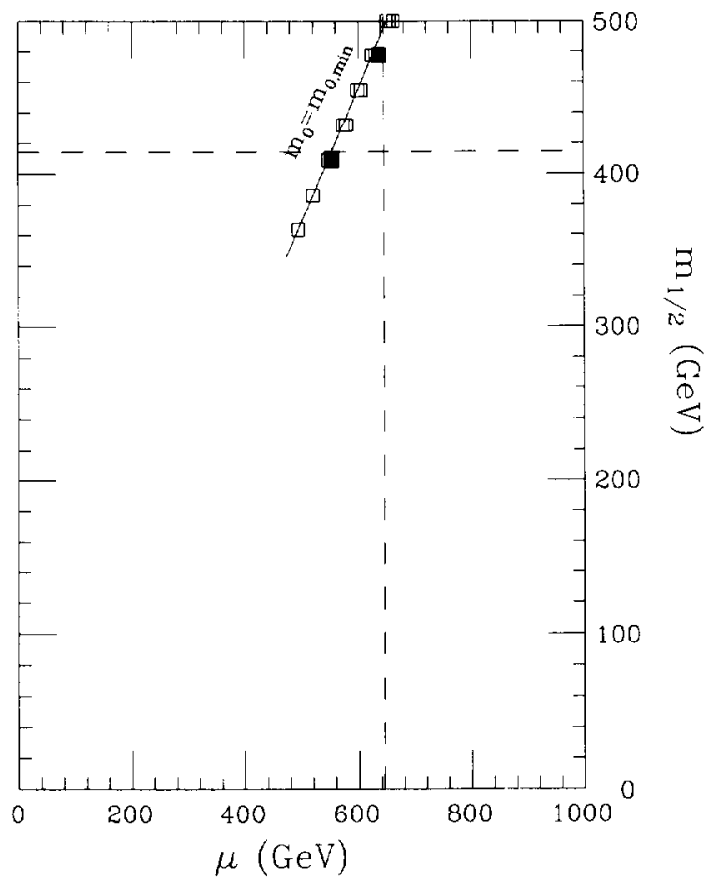

Fig. 12. (a) Parameter space in the $\left(m_{1 / 2}, m_{0}\right)$ plane for $\tan \beta=53, \delta_{1}=0$ and $\delta_{2}=0$. Filled squares denote configurations excluded by direct detection with a Ge detector [39]. Other notations are as in Fig. 9. (b) Parameter space represented in the $\left(m_{1 / 2}, \mu\right)$ plane. Notations are the same as in (a).

$h, H$ are dominant compared to $A$. The low-energy neutralino-quark effective Lagrangian generated by Higgs exchange may be written as follows [35]

$$
\mathcal{L}_{\mathrm{eff}}=\sqrt{2} G_{F} \frac{m_{Z}}{m_{h, H}^{2}} F_{h, H} \sum_{q} k_{q} m_{q} \bar{\psi}_{\chi} \psi_{\chi} \bar{q} q
$$

Here $F_{h, H}$ is the ratio of the Higgs-neutralino coupling to the $S U(2)$ gauge coupling, which depends on the composition of $\chi$

$$
F_{h}=a_{2}\left(a_{3} \sin \alpha+a_{4} \cos \alpha\right), \quad F_{H}=a_{2}\left(a_{3} \cos \alpha-a_{4} \sin \alpha\right)
$$

and the $k_{q}$ are given, for the up-type quarks and the down-type quarks respectively, by

$$
\begin{array}{ccc} 
& H & h \\
k_{u} & \sin \alpha / \sin \beta & \cos \alpha / \sin \beta \\
k_{d} & \cos \alpha / \cos \beta & -\sin \alpha / \cos \beta .
\end{array}
$$

Note that, in general, since $\tan \beta>1$, the strength of the coupling to the down-type quarks is bigger than the one to the up-type quarks, and $\mathcal{L}_{\text {eff }}$ usually gets a sizeable contribution when the $h$ boson is exchanged ( $h$ is lighter than $H$ and is therefore favored because of the propagator denominator in Eq. (19)) and when $\alpha \simeq \pi / 2$, i.e., when $M_{A} \lesssim O\left(M_{Z}\right)$. When this regime does not apply, the size of $\mathcal{L}_{\text {eff }}$ is much suppressed.

The cross section for elastic neutralino-nucleus scattering which follows from the effective Lagrangian (19) will be given in Section 9.2. 
(a)

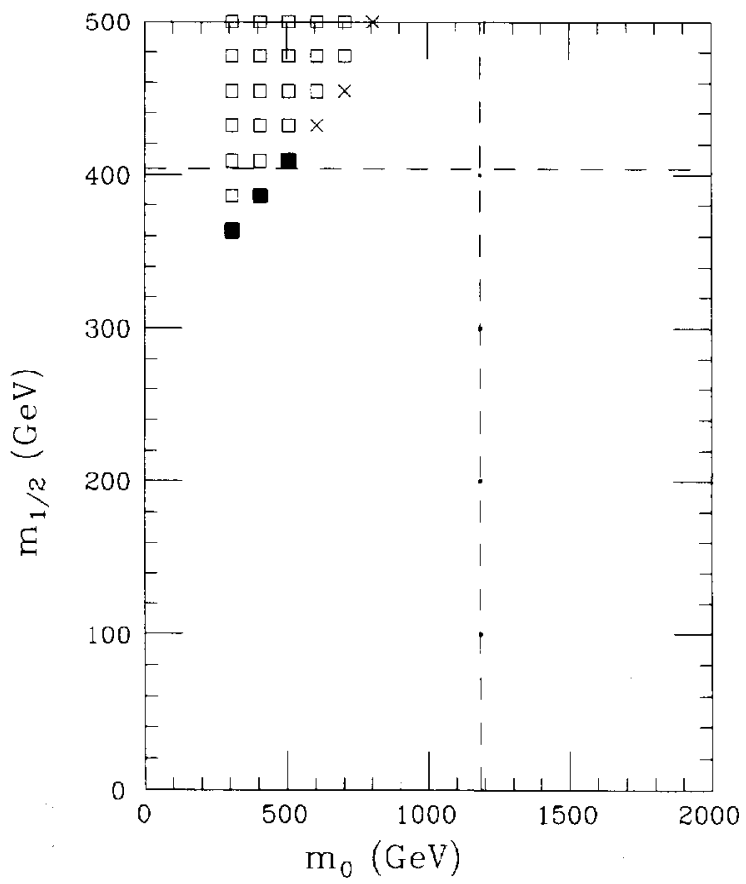

(b)

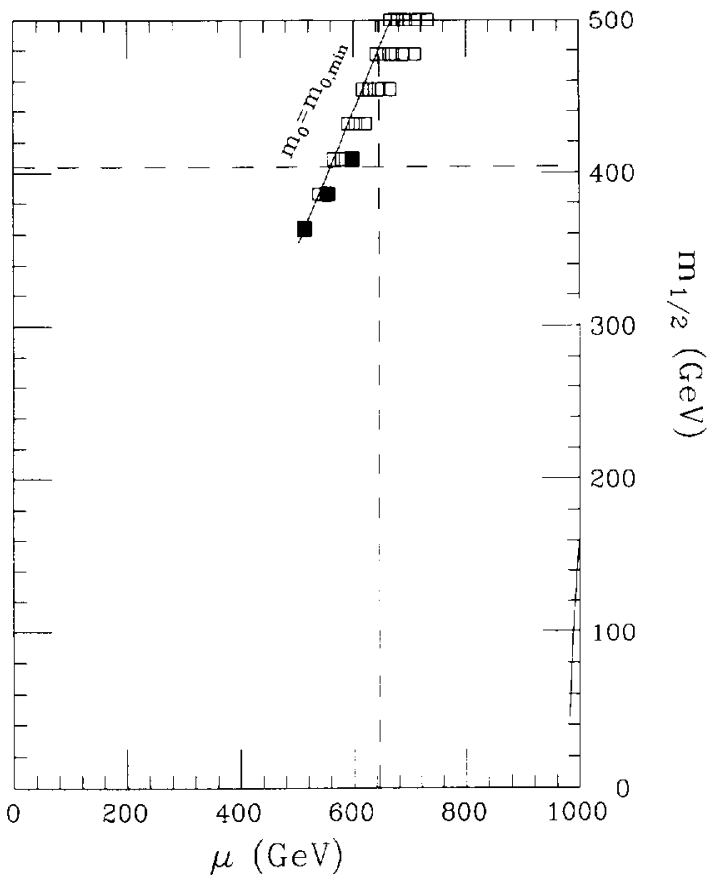

Fig. 13. The same as in Fig. 12, but with $\delta_{1}=0$ and $\delta_{2}=-0.3$.

(a)

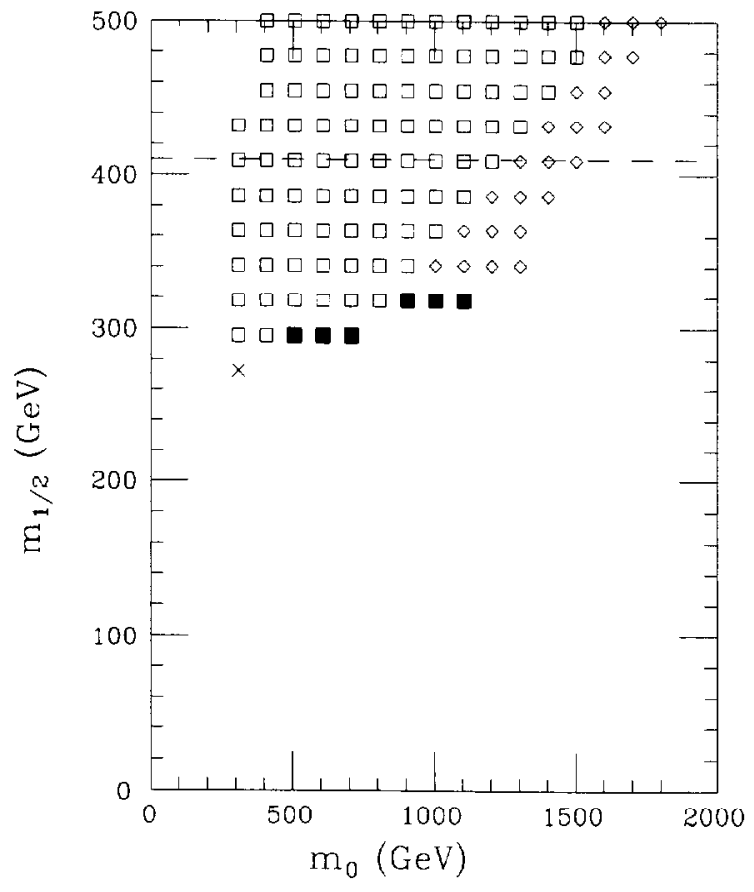

(b)

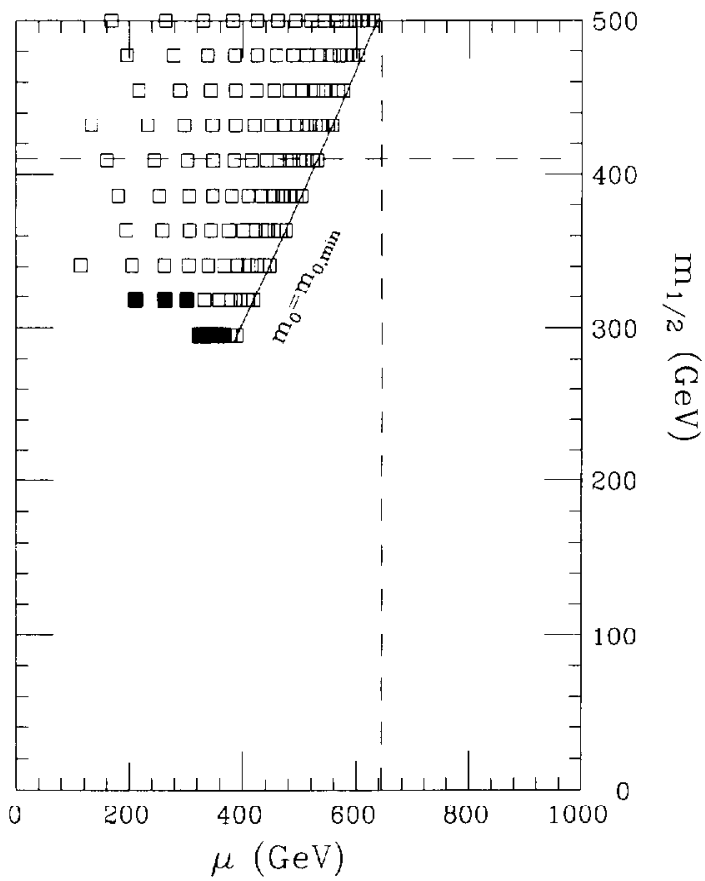

Fig. 14. The same as in Fig. 12, but with $\delta_{1}=0.7$ and $\delta_{2}=0.4$. 
(a)

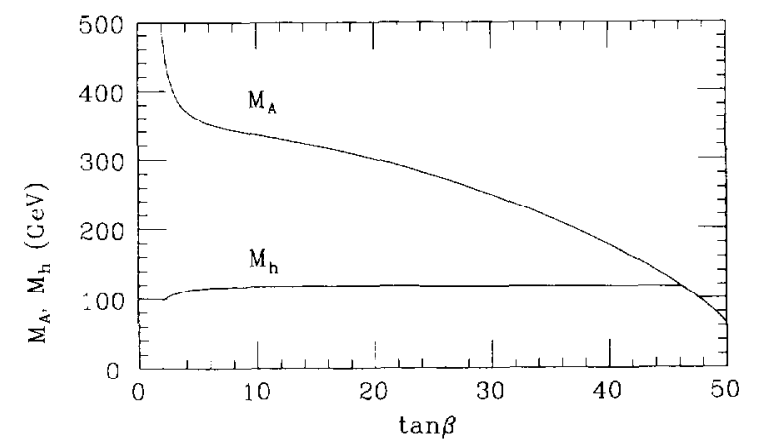

(b)

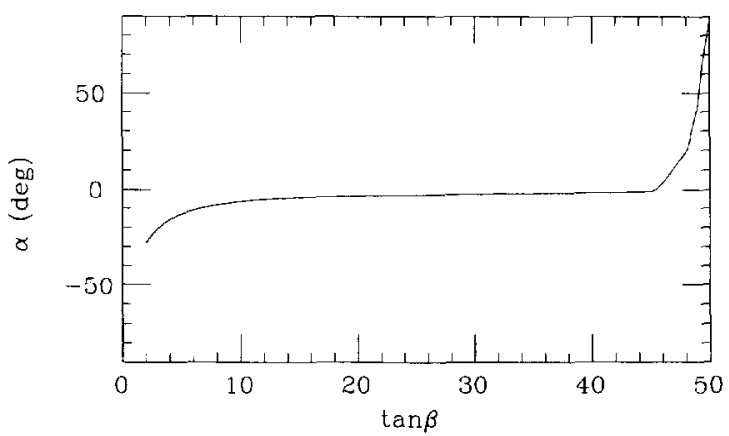

Fig. 15. (a) Graphs of $M_{A}$ and $M_{h}$ as functions of $\tan \beta$. (b) Mixing angle $\alpha$ as a function of $\tan \beta$. Here the representative point is $m_{t)}=100 \mathrm{GeV}, m_{1 / 2}=200 \mathrm{GeV}$.

\section{Direct detection}

Much experimental activity is under way in the direct search for neutralino dark matter and the perspectives for significant improvements in experimental sensitivities are encouraging [36]. In this class of experiments, a relic neutralino would be detected by the amount of energy released by its elastic scattering off nuclei in an appropriate apparatus. A signature would be provided by a yearly modulation of the signal, whose observations would require high statistics and extremely good stability in the detector response. Here we evaluate the event rates for this process extending previous analyses to the non-universal $\delta_{i} \neq 0$ case. Various materials are being used in the current experiments and others are under investigation for future detectors. In this paper we analyse two of the most interesting materials: Ge (in its natural composition) [37-39] and ${ }^{129} \mathrm{Xe}[40]$.

\subsection{Differential rates}

The nuclear recoil spectrum may be evaluated from the expression

$$
\frac{d R}{d E_{R}}=\sum_{i} \frac{R_{0, i}}{\left\langle E_{R}^{\max }\right\rangle} F_{i}^{2}\left(E_{R}\right) I\left(E_{R}\right)
$$

where

$$
R_{0, i}=N_{T} \frac{\rho_{\chi}}{m_{\chi}} \sigma_{i}\langle v\rangle
$$

In Eqs.(22)-(23) we use the following notations: the subscript $i$ refers to the two cases of coherent and spindependent effective interactions, $N_{T}$ is the number of the target nuclei per unit of mass, $\rho_{X}$ is the local neutralino matter density, and $E_{R}$ is the nuclear recoil energy given by $E_{R}=m_{r e d}^{2} v^{2}\left(1-\cos \theta^{*}\right) / m_{N}$, where $\theta^{*}$ is the scattering angle in the neutralino-nucleus center-of-mass frame, $m_{N}$ is the nuclear mass, $m_{\mathrm{red}}$ is the neutralinonucleus reduced mass and $v$ is the relative velocity. The maximum value of $E_{R}$ is $E_{R}^{\max }=2 \mathrm{~m}_{\mathrm{red}}^{2} v^{2} / \mathrm{m}_{N}$. Returning to (22)-(23), $F\left(E_{R}\right)$ denotes the nuclear form factor, and $\sigma_{i}$ is the (coherent/spin-dependent) neutralino-nucleus cross section. The factor $I\left(E_{R}\right)$ is given by

$$
I\left(E_{R}\right)=\frac{\left\langle v^{2}\right\rangle}{\langle v\rangle} \int_{l^{\prime \min }\left(E_{R}\right)}^{i^{\prime m a x}} d v \frac{f(v)}{v}
$$


where $f(v)$ is the velocity distribution of neutralinos in the Galaxy, as measured in the Earth's rest frame, and $v_{\min }\left(E_{R}\right)$ is given by $v_{\min }\left(E_{R}\right)=\left(m_{N} E_{R} /\left(2 m_{\text {red }}^{2}\right)\right)^{1 / 2}$. The averages appearing in Eqs. (22)-(24) denote averages over the velocity distribution in the Earth's rest frame. An explicit formula for $I\left(E_{R}\right)$ in the case of a Maxwellian velocity distribution may be found in Ref. [4].

The differential rates to be discussed below will be expressed in terms of the electron-equivalent energy $E_{e e}$ rather than in terms of $E_{R}$. These two variables are proportional: $E_{\ell e}=Q E_{R}$ where $Q$ is called the quenching factor: typical values of $Q$ will be discussed shortly.

\subsection{Neutralino-nucleus elastic cross sections}

The total cross sections for neutralino-nucleus elastic scattering have been evaluated following standard procedures $[3,4,35,41,42]$. Here we only summarize some of the main properties. Neutralino-quark scattering is described by amplitudes with Higgs-boson exchanges and $Z$-boson exchange in the t-channel, and by amplitudes with squark exchanges in the s- and u-channels. The neutral Higgs bosons considered here are the two CP-even busons: $h, H$ and the CP-odd one: $A$, whose couplings were previously discussed in Section 8.

The relevant properties for these amplitudes are: (1) Higgs-boson exchanges contribute a coherent cross section which vanishes only when there is no zino-higgsino mixture in the neutralino composition [35], (2) $Z$-boson exchange provides a spin-dependent cross section which receives contributions only from the higgsino components of $\chi$, (3) squark exchanges contribute a coherent cross section (due to zino-higgsino mixing) as well as a spin-dependent cross section (due mainly to the gaugino components of $\chi$ ) [41]. As examples we recall here only the expressions for the coherent cross section due to the exchange of a Higgs boson ( $h$ or $H$ ) and the spin-dependent one due to $\mathrm{Z}$ exchange.

The former cross section is casily evaluated from the effective Lagrangian of Eq. (19) [35]

$$
\sigma_{\mathrm{CH}}=\frac{8 G_{F}^{2}}{\pi} \frac{m_{Z}^{2}}{m_{h, H}^{4}} \alpha_{h, H}^{2} m_{r e d}^{2} A^{2}
$$

where $A$ is the nuclear mass number and $\alpha_{h, H}$ is given by

$$
\alpha_{h . H}=F_{h, H} I, \quad I=\sum_{q} k_{q} m_{q}\langle N|\bar{q} q| N\rangle .
$$

The quantity I may be expressed conveniently in terms of the $\pi N$ sigma-term $\sigma_{\pi N}$ and of a parameter $a$ which is related to the strange-quark content of the nucleon $y$ by

$$
a=y\left(m_{s} /\left(m_{u}+m_{d}\right)\right), \quad y=2 \frac{\langle N|\bar{s} s| N\rangle}{\langle N|\bar{u} u+\bar{d} d| N\rangle} .
$$

One has

$$
I \simeq k_{u} g_{u}+k_{d} g_{d}
$$

where

$$
g_{u}=\frac{4}{27}\left(m_{N}+\frac{19}{8} \sigma_{\pi N}-a \sigma_{\pi N}\right), \quad g_{d}=\frac{2}{27}\left(m_{N}+\frac{23}{4} \sigma_{\pi N}+\frac{25}{2} a \sigma_{\pi N}\right) .
$$

Unfortunately, the values of both the quantities $y$ and $\sigma_{\pi N}$ are somewhat uncertain. Here, for $y$ we use the central value of the most recent evaluation: $y=0.33 \pm 0.09$, obtained from a lattice calculation [43]. For $\sigma_{\pi N}$, which is derived by phase-shift analysis and dispersion relation techniques from low-energy pion-nucleon scattering cross-sections [44,45], we employ the value of Ref. [45]: $\sigma_{\pi N}=45 \mathrm{MeV}$. We then find the results: $g_{u}=123 \mathrm{MeV}, g_{d l}=288 \mathrm{MeV}$ (we use $2\left(m_{s} /\left(m_{u}+m_{d}\right)\right)=29[46]$ ). We note that these values further reinforce the role of the down-type quarks as compared to the up-type ones. 
Table 1

Characteristics of some current experiments. In the second column is reported the quenching factor $Q$, in the third column the electronequivalent energy at threshold, in the fourth the square of the form factor at threshold, and in the last column the present experimental sensitivity.

\begin{tabular}{lllll}
\hline Nucleus & $Q$ & $E_{e e}^{t h}(\mathrm{keV})$ & $F^{2}\left(E_{R}^{t / h}\right)$ & evts $(\mathrm{kg} \mathrm{d} \mathrm{keV})$ \\
\hline Ge $|38|$ & 0.25 & 2 & 0.87 & 3.0 \\
Ge $|39|$ & 0.25 & 12 & 0.41 & 0.2 \\
Xe $|40|$ & 0.80 & 40 & 0.07 & 0.8 \\
\hline
\end{tabular}

Wc point out that the Higgs-nucleon couplings for nucleons bound in a nucleus may be renormalized by the nuclear medium. As a consequence, the strength of $I$ might in principle be reduced to some extent [47]. However, this effect is neglected herc.

Now let us turn to the spin-dependent cross section due to $\mathrm{Z}$ exchange. This may be cast into the usual form [41,42]

$$
\sigma_{\mathrm{SD}}=\frac{8 G_{F}^{2}}{\pi}\left(a_{3}^{2}-a_{4}^{2}\right)^{2} m_{r e d}^{2}\left(\sum_{q} T_{3 L, q} \Delta q\right)^{2} \lambda^{2} J(J+1) .
$$

In this paper we use this formula for ${ }^{73} \mathrm{Ge}$ (this isotope is present at the level of $7.8 \%$ in the natural composition of $\mathrm{Ge}$ ) and to ${ }^{129} \mathrm{Xe}$. For these nuclei we employ the values of $\lambda^{2}$ obtained in the odd-group model [3], where only the odd nuclear species in odd-even nuclei are explicitly taken into account. The $\Delta q$ 's in Eq. (30) denote the fractions of the nucleon spin carried by the quarks $q$ in the nucleon of the odd species, and the $T_{3 L, q}$ 's stand for the third components of the quark weak isospin. The values for the $\Delta q$ 's are taken from Ref. [48].

It is worth noticing that the event rates for neutralino direct detection with the materials considered here are largely dominated by coherent effects in most regions of the parameter space. In the small domains where spindependent effects dominate over the coherent ones the total rates are usually too small to allow detection. The experimental strategy of employing materials enriched in heavy isotopes of high spin is interesting for a search for hypothetical dark matter particles which interact with matter via substantial spin-dependent interactions. However, this approach does not appear to be very fruitful for neutralinos.

One more ingredient which enters the event rate in Eq. (22) is the nuclear form factor, which depends sensitively on the nature of the effective interaction involved in the neutralino-nucleus scattering. For the coherent case, we simply employ the standard parameterization [49]

$$
F\left(E_{R}\right)=3 \frac{j_{1}\left(q r_{0}\right)}{q r_{0}} e^{-1 / 2} s^{2} q^{2}
$$

where $q^{2} \equiv|q|^{2}=2 m_{N} E_{R}$ is the squared three-momentum transfer, $s \simeq 1 \mathrm{fm}$ is the thickness parameter for the nucleus surface, $r_{0}=\left(r^{2}-5 s^{2}\right)^{1 / 2}, r=1.2 A^{1 / 3} \mathrm{fm}$ and $j_{1}\left(q r_{0}\right)$ is the spherical Bessel function of index 1 .

The form factor in Eq. (31) introduces a substantial suppression in the recoil spectrum unless $q r_{0} \ll 1$. A noticeable reduction in $d R / d E_{R}$ may already occur at threshold $E_{R}=E_{R}^{\text {th }}=E_{\mathrm{ee}}^{\mathrm{th}} / Q$ when $r_{0} \sqrt{2 m_{N} E_{R}^{\mathrm{th}}}$ is not small compared to unity. The actual occurrence of this feature depends on parameters of the detector material: nuclear radius, quenching factor, threshold energy $E_{\mathrm{ee}}^{\text {th }}$. The values of these parameters for the nuclei considered in this paper are reported in Table $1[36,38-40]$, and the values of $F^{2}\left(E_{R}^{\text {th }}\right)$ calculated from Eq. (31) are given in the same table. Since we consider in this paper mainly the value of the differential rate near threshold, $F^{2}\left(E_{R}^{\text {th }}\right)$ is the most relevant quantity. We see from the values in Table 1 that the reduction introduced by the form factor is moderate in $\mathrm{Ge}$, but quite substantial in ${ }^{129} \mathrm{Xe}$.

In general, for the spin-dependent case there are no analytic expressions for the form factors. However, numerical analyses have been performed for a number of nuclei. The general feature is that these form factors 
have a much milder dependence on $E_{R}$ as compared to the coherent ones, because only a few nucleons participate in the neutralino-nucleus scattering in this case. In our evaluations we use the results of Refs. $[49,50]$ for ${ }^{131} \mathrm{Xe}$ and ${ }^{73} \mathrm{Ge}$ respectively.

\subsection{Local neutralino density}

We denote the local halo density by $\rho_{l}$, for which we use the estimate $\rho_{l}=0.5 \mathrm{GeV} \mathrm{cm}^{-3}$ [51]. For the value of the local neutralino density $\rho_{X}$ to be used in the rate of Eq. (23), for each point of the model parameter space we take into account the relevant value of the cosmological neutralino relic density. When $\Omega_{X} h^{2}$ is larger than a minimal $\left(\Omega h^{2}\right)_{\min }$ required by observational data and by large scale structure calculations we simply put $\rho_{\chi}=\rho_{l}$. When $\Omega_{X} h^{2}$ turns out less than $\left(\Omega h^{2}\right)_{\min }$, the neutralino may only provide a fractional contribution $\Omega_{\chi} h^{2} /\left(\Omega h^{2}\right)_{\min } \equiv \xi$ to $\Omega h^{2}$; in this case we take $\rho_{\chi}=\rho_{l} \xi$. The value to be assigned to $\left(\Omega h^{2}\right)_{\min }$ is somewhat arbitrary. Here we set it equal to 0.1 .

It is worth remarking here that, due to this scaling procedure, for the direct detection rate one has: (i) $R_{0, i} \propto \rho_{l} \sigma_{i}$ for $\Omega_{\chi} h^{2} \geq\left(\Omega h^{2}\right)_{\min }$ and (ii) $R_{0, i} \propto \rho_{l} \xi \sigma_{i} \propto \rho_{l} \sigma_{i} /\left\langle\sigma_{\mathrm{ann}} v\right\rangle_{\text {int }}$ for $\Omega_{\chi} h^{2}<\left(\Omega h^{2}\right)_{\min }$. Thus the rate $R_{0, i}$ is large in the regions of the parameter space where $\sigma_{i}$ is large. This is trivial in case (i), but it is also true in case (ii), since when $\sigma_{i}$ is large also $\sigma_{\text {ann }}$ increases but in such a way that usually the ratio $\sigma_{i} / \sigma_{\text {ann }}$ increases too. Because of the relation $\Omega_{\chi} h^{2} \propto\left\langle\sigma_{\mathrm{ann}} v\right\rangle_{\mathrm{int}}^{-1}$ it follows that $R_{0, i}$ is large for neutralino configurations with modest values of the relic abundance, and vice versa.

\subsection{Results for detection rates}

The most significant quantity in comparing experimental data and theoretical evaluations for direct detection is the differential rate $d R / d E_{e e}=\left(d R / d E_{R}\right) / Q$ (with $d R / d E_{R}$ defined in Eq. (22)) rather than the total rates, obtained by integration over wide ranges of $E_{e e}$. By using the differential rate instead of the integrated ones, one obtains the best signal-to-background ratio. Note that the experimental spectra, apart from an energy interval around threshold, usually show a very flat behaviour, whereas signals for light neutralinos are decreasing functions of the nuclear recoil energy.

A complete procedure would then be to compare the experimental and theoretical rates over the whole $E_{e e}$ range. However, to simplify the presentation here, we give our results in terms of the rate integrated over a narrow range of $1 \mathrm{keV}$ at a specific value of $E_{e e}$, the one which appears the most appropriate for each experiment: typically it corresponds to a point close to the experimental threshold. To be definite we consider the following cases:

(i) Ge (natural composition). Among the various running experiments [36], we select the two which, at present, appear to provide the most stringent limits: (a) Caltech-PSI-Neuchatel [38] with $E_{e e}^{\text {th }}=2 \mathrm{keV}$, differential rate $\simeq 3$ events $/\left(\mathrm{kg}\right.$ day $\mathrm{keV}$ ); (b) Heidelberg-Moscow [39] with $E_{e e}^{\text {th }}=12 \mathrm{keV}$, differential rate $\simeq 0.2$ events $/(\mathrm{kg}$ day $\mathrm{keV})$. Correspondingly, for $\mathrm{Ge}$ we have evaluated our rate by integrating $d R / d E_{\text {ee }}$ over the range (2-3) $\mathrm{keV}$ for experiment (a) and over (12-13) $\mathrm{keV}$ for experiment (b). It turns out that the case (b) provides the most stringent bound also for light neutralinos

(ii) ${ }^{129} \mathrm{Xe}$. In this case, taking into account the features of the DAMA experiment [40], we have considered the rate $R$ integrated over the range $40-41 \mathrm{keV}$.

Our results are shown in Figs. 16-19. Figs. 16-18 report the rate for a Ge detector for the regions of the parameter space which are depicted in Figs. 12-14, respectively. In parts (a) and (b) of each figure, $R$ is displayed in the form of a scatter plot, in terms of $m_{\chi}$ and of the relic abundance, respectively. The horizontal line denotes the present level of sensitivity in the Heidelberg-Moscow experiment. We notice that, in all cases shown in these figures, the experimental sensitivity is already, for some configurations, at the level of the predicted rate. Some points of the supersymmetric parameter space, denoted by filled squares in Figs. 12-14, are even already excluded by present data. The exploration potential of this class of experiments as the 
(a)

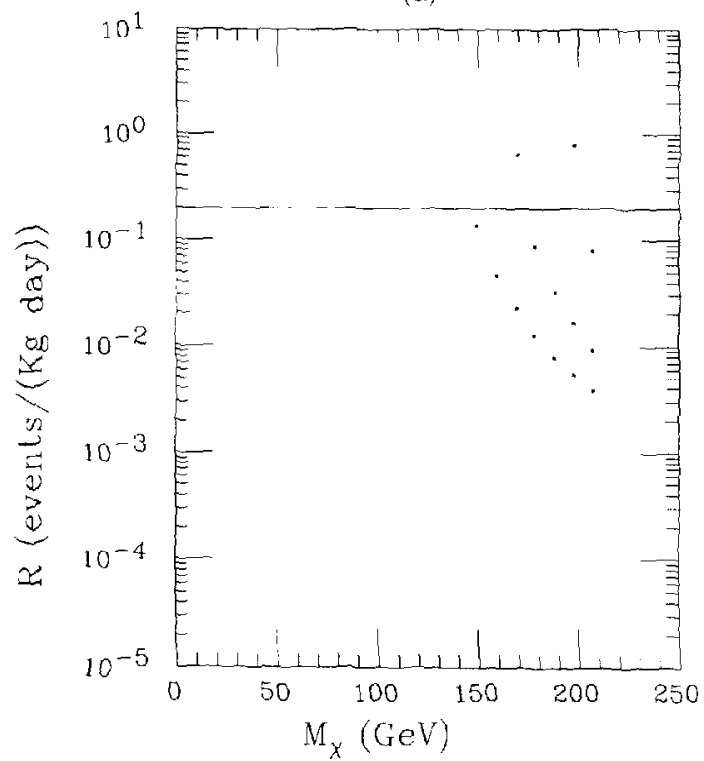

(b)

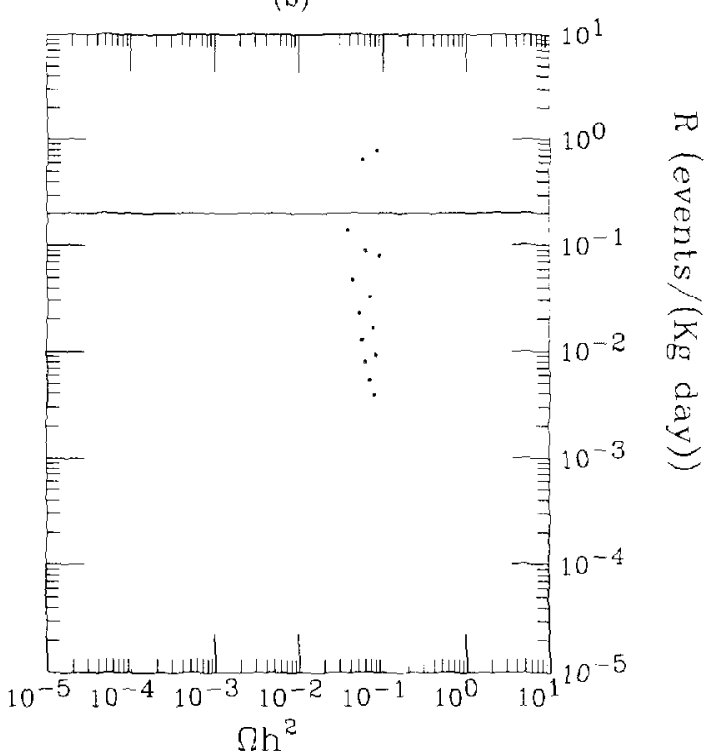

Fig. 16. Scatter plot of the rate for direct detection with a $G e[39]$ detector for $\tan \beta=53, \delta_{1}=0$ and $\delta_{2}=0$, as a function of $m{ }_{\chi}$ (a) and as a function of $\Omega_{\chi} h^{2}$ (b). Parameters are varied on a linear equally-spaced grid over the ranges: $10 \mathrm{GeV} \leq m_{0} \leq 2$ TeV, $4.5 \mathrm{GeV} \leq m_{1 / 2} \leq 500 \mathrm{GeV}$

(a)

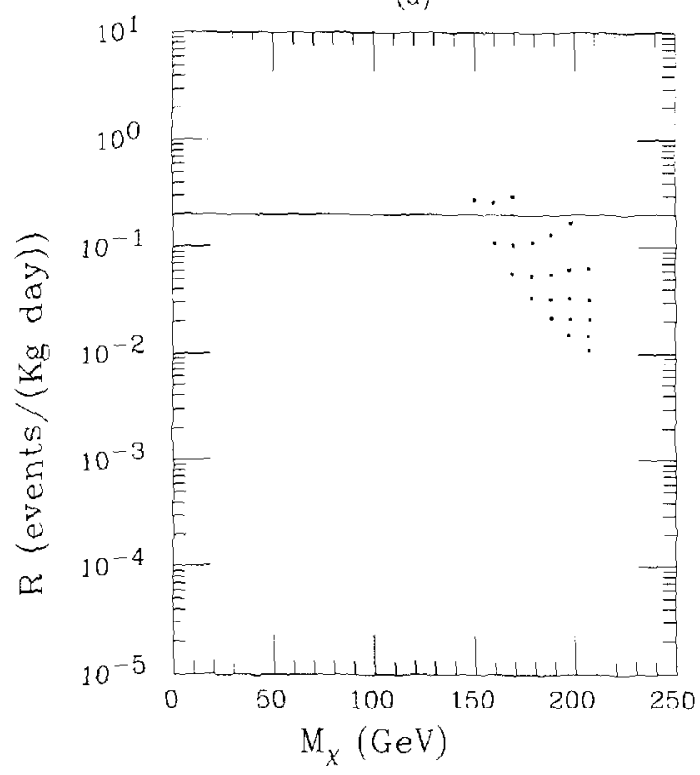

(b)

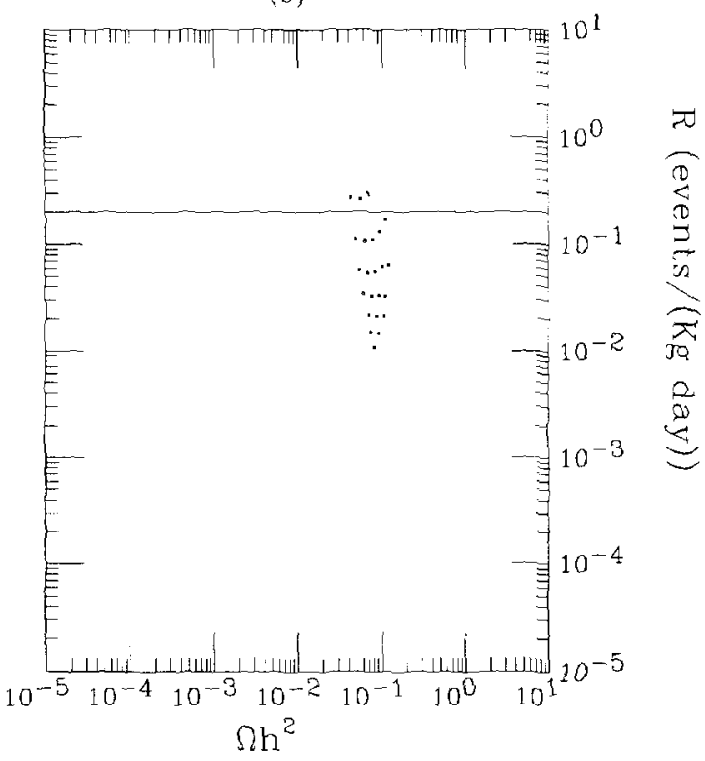

Fig. 17. The same as in Fig. 16, but with $\delta_{1}=0$ and $\delta_{2}=-0.3$.

sensitivity is improved is apparent from these figures. Fig. 19 shows the rate $R$ for ${ }^{129} \mathrm{Xe}$ for the region of the parameter space displayed in Fig. 13: again the horizontal line gives the present experimental sensitivity. A comparison of Fig. 19 with Fig. 17 shows that the Ge experiments are currently more effective. However, it has to be noticed that experiments with liquid Xe may become extremely competitive in the future [40]. 
(a)

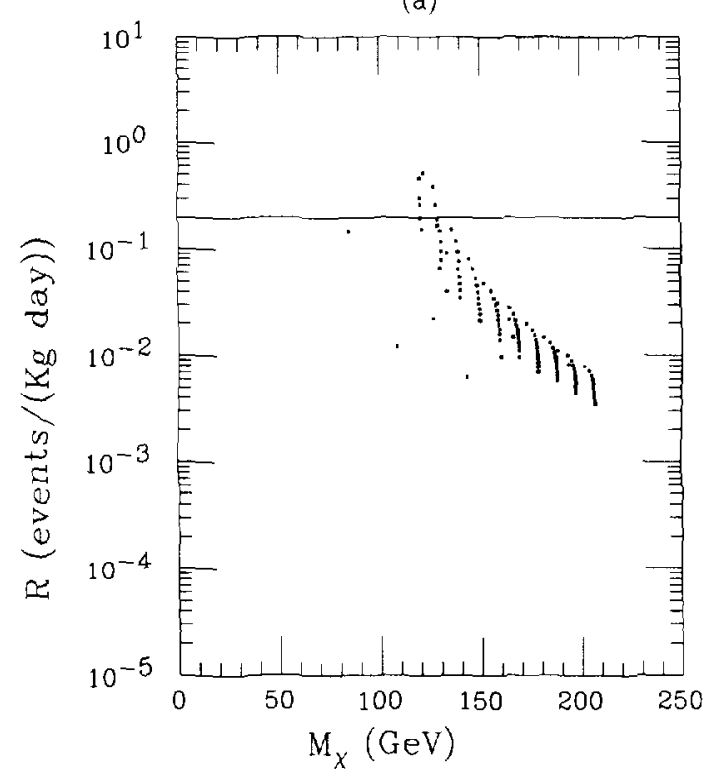

(b)

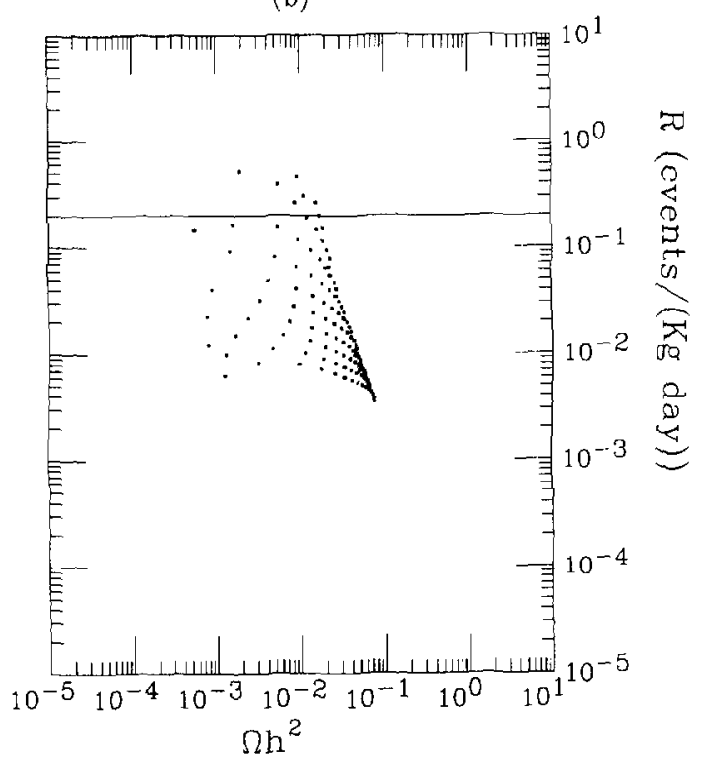

Fig. 18. The same as in Fig. 16, but with $\delta_{1}=0.7$ and $\delta_{2}=0.4$.

(a)

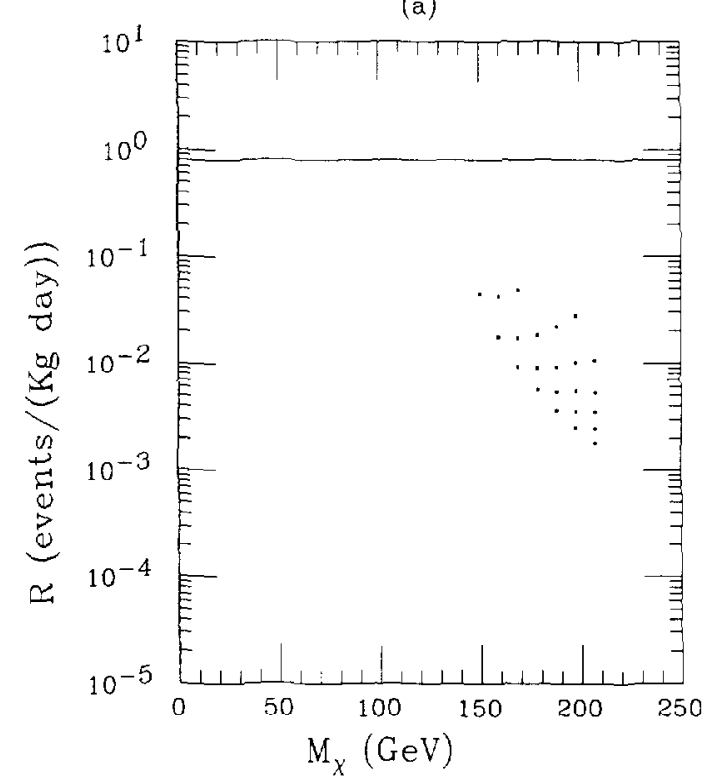

(b)

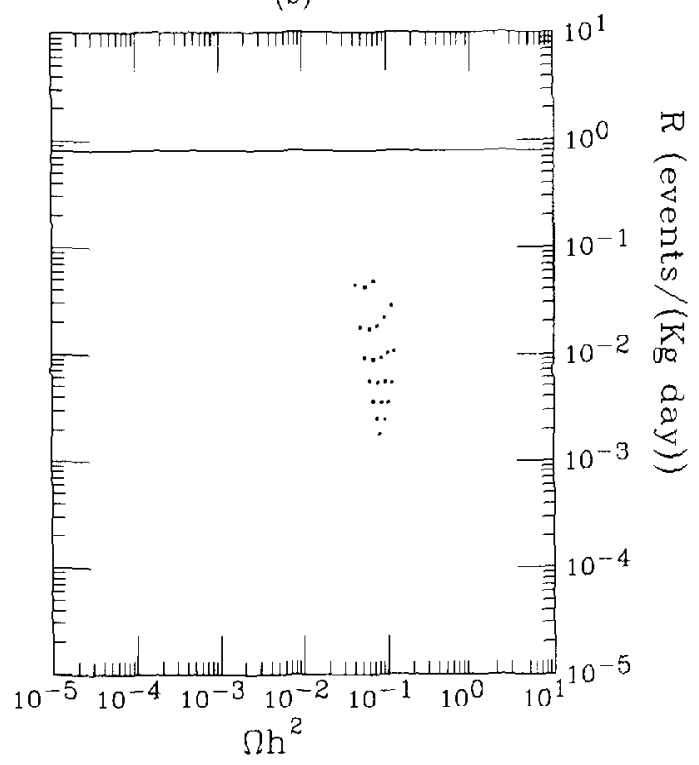

Fig. 19. Scatter plot of the rate for direct detection with a $X e$ [40] detector for $\tan \beta=53, \delta_{1}=0$ and $\delta_{2}=-0.3$, as a function of $m_{\chi}$ (a) and as a function of $\Omega_{\chi} h^{2}$ (b). Parameters are varied on a linear equally-spaced grid over the ranges: $10 \mathrm{GeV} \leq m_{0} \leq 2 \mathrm{TeV}$, $45 \mathrm{GreV} \leq m_{1 / 2} \leq 500 \mathrm{GeV}$.

A few more remarks are in order here:

(i) The cases displayed in Figs. 16-18 present the common feature of providing fair chances for direct detection. This is not a surprise, since these representative points all belong to the category of configurations with small values of $M_{A}$. As was stressed before, once we move away from these appealing physical 
regions of the neutralino parameter space, the rates for direct detection may fall far below (by many orders of magnitude) the detection sensitivities (present or future). This unfortunate situation occurs, for instance, typically as we move towards smaller values of $\tan \beta$. However, one should keep in mind that the regime of very large $\tan \beta$, where signals may be sizeable, represents a very interesting scenario, deserving much attention and exploration. In fact this is one of the two options, very small or very large $\tan \beta$, which seem to fit low-energy phenomenology at the best [52].

(ii) The scatter plots in parts (b) of Figs. 16-19 show explicitly a property previously mentioned in Section 9.3 , namely that the scaling procedure adopted to evaluate the neutralino local density implies a $R-\Omega_{\chi} h^{2}$ correlation. Configurations which provide a measurable $R$ usually entail a low $\Omega$ and viceversa. Only in a few cases the neutralino may be detectable by direct detection and also provide a sizeable contribution to $\Omega$.

\section{Conclusions}

In the present paper we have discussed some possible scenarios for neutralino dark matter which originate from the relaxation of the assumption of strict universality for soft scalar masses at $M_{G U T}$.

This approach derives from the general consideration that many crucial theoretical points entering not only grand unified and supersymmetric theories, not to mention the Standard Model, are far from being understood and/or verified. For this reason, any new theoretical assumption has to be fully scrutinized. This is even more important because new assumptions in supersymmetric models are often introduced not because of solid arguments, but rather for the sake of simplicity and for the need to reduce the large number of free parameters that would otherwise prevent any firm prediction.

In our work we have discussed different scenarios, by considering various physical constraints in a sort of hierarchical order, giving top priority to the requirement of radiative EWSB, implemented with a no-fine-tuning criterion, and to the cosmological relic neutralino density constraint. Some other assumptions, often introduced in the literature, have been relaxed in our work. This is in particular the case for universality in the soft scalar masses. However, it has to be remarked that the type of departure from universality that we have considered in our paper is far from being the most general one, as was noticed in Section 2. In particular, it only refers to the Higgs masses, and not to the sfermion masses.

The implications of the various scenarios on neutralino relic abundances and rates for detection rates have been analysed, and the impact of a non-universality in $m_{0}$ has been discussed for the whole range of $\tan \beta$. We have shown that the departure from $m_{0}$ universality is particularly interesting in two respects:

(i) Small values of $M_{A}$ are allowed: this has in itself the dramatic consequence for direct detection of generating a large value for the angle $\alpha$ and large couplings to matter of the lightest neutralino $\chi$.

(ii) Higgsino or mixed higgsino-gaugino configurations appear for all $\tan \beta$ : this contrasts with the pure gaugino configurations favoured by strict $m_{0}$ universality. Consequences of such a departure from universality on the size of the neutralino relic abundance have been analysed for both large and small values of $\tan \beta$. It has been shown that, because of the previous properties, deviations from universality may reduce the value of $\Omega_{\chi} h^{2}$.

The predicted rates for direct detection have been analysed in detail and compared with current and foreseen experimental sensitivities. The role of the previous properties in opening interesting perspectives for this kind of search has been elucidated. We find that presently-running experiments are already impacting interesting regions of the neutralino parameters space in some of the non-universal scenarios discussed here. 


\section{Acknowledgements}

We wish to express our thanks to Uri Sarid for interesting discussions and for contributions in the very early stages of our work. We also gratefully acknowledge very useful conversations with Marek Olechowski and informative discussions on experimental aspects of direct detection with Pierluigi Belli, Rita Bernabei and Luigi Mosca. NF wishes to express his gratitude to the Fondazione A. Della Riccia for a fellowship. This work was supported in part by the Ministero della Ricerca Scientifica e Tecnologica (Italy). Partial financial support was provided by the Theoretical Astroparticle Network under contract No. CHRX-CT93-0120 of the Direction General of the EEC.

\section{References}

I1 H. P. Nilles, Phys. Rep. 110 (1984) 1; H. E. Haber and G. L. Kane, Phys. Rep. 117 (1985) 75; R. Barbieri Riv. Nuovo Cim. II (1988) I.

12] A comprehensive list of the theoretical references on this topic would be too long to be reported here. Only some of the most recent papers are given in $|3,4|$, where also references to previous works may be found. The same applies to references in |5-7|. For reviews on detection methods for dark matter candidates, see J. R. Primack, D. Seckel and B. Saroulet, Ann. Rev. Nucl. Part. Sci. 38 (1988) 751, and P. F. Smith and J. D. Lewin, Phys. Rep. 187 (1990) 203. An up-dated review of the phenomenology of neutralino dark matter is presented in G. Jungman, M. Kamionkowski and K. Griest, Phys. Rep. 267 (1996) 195.

|3| J. Ellis and R. Flores, Phys. Lett. B263 (1991) 259; Nucl. Phys. B400 (1993) 25; Phys. Lett. B300 (1993) 175

I4| A. Bottino, V.de Alfaro, N. Fornengo, G. Mignola, S. Scopel, and C. Bacci et al. (BRS Collaboration), Phys. Lett. B295 (1992) 330: A. Bottino, V. de Alfaro, N. Fornengo, G. Mignola and S. Scopel, Astroparticle Physics 2 (1994) 77.

15| G. Gelmini, P. Gondolo and E. Roulet, Nucl. Phys. B351 (1991) 623; M. Kamionkowski, Phys. Rev. D44 (1991) 3021; F. Halzen, T. Stelzer and M. Kamionkowski Phys. Rev. D45 (1992) 4439 ; M. Mori et al., Phys. Rev D48 (1993) 5505; V. Berezinsky, TAUP93 Proceedings, Nucl. Phys. B (Proc. Suppl) 35 (1994) 484.

16| A. Bottino, N. Fornengo, G. Mignola and L. Moscoso, Astroparticle Physics 3 (1995) 65.

|7| J. Ellis, R. A. Flores, K. Freese, S. Ritz, D. Seckel and J. Silk, Phys. Lett. B214 (1988) 403; M. Kamionkowski and M. Turner, Phys. Rev. D43 (1991) 1774; V. Berezinsky, A. Bottino and G. Mignola, Phys. Lett. B325 (1994) 136; L. Bergström and J. Kaplan, Astroparticle Physics 2 (1994) 261:

G. Jungman and M. Kamionkowski, Phys. Rev. D49 (1994) 2316; Phys. Rev. D51 (1995) 3121; A. Bottino, C. Favero, N. Fornengo, and G. Mignola, Astroparticle Physics 3 (1995) 77.

|8| See, for instance, H. Baer et al., Florida State University preprint FSU-HEP-950401, hep-ph/9503479 (1995).

19| L. E. Ibañez and C. Lopez, Nucl. Phys. B233 (1984) 511;

J. Ellis and F. Zwirner, Nucl. Phys. B338 (1990) 317; J. Ellis and L. Roszkowski, Phys. Lett. B283 (1992) 252; M. Drees and M. M. Nojiri, Nucl. Phys. B369 (1992) 54; M. Olechowski and S. Pokorski, Nucl. Phys. B404 (1993) 590; R.G. Roberts and L. Roszkowski, Phys. Lett. B309 (1993) 329; R. Arnowitt and P. Nath, Texas A\&M University preprint CTP-TAMU-52-93, hepph/9309277 (1993); J. L. Lopez, D.V. Nanopoulos and A. Zichichi, Riv. Nuovo Cim. 17 (1994) 1; W. de Boer, R. Ehrel and D.I. Kazakov, Karlsruhe University preprint IEKP-KA/94-04 (1994); P. Nath and R. Arnowitt, CERN preprint CERN-TH-7363-94 (1994): G. L. Kane, C. Kolda, L. Roszkowski and J. D. Wells, Phys. Rev. D49 (1994) 6173; R. Rattazzi and U. Sarid, Stanford University preprint SU-ITP-94-16, (1995); X. Wang, J. Lopez and D. Nanopoulas, Phys. Lett. B348 (1995) 105.

| $10 \mid$ V. Barger, M. S. Berger and P. Ohmann, Phys. Rev. D49 (1994) 4908.

1 11 E. Diehl, G. L. Kane, C. Kolda and J. D. Wells, Michigan University preprint UM-TH-94-38, hep-ph/9502399 (1995).

[12] Neutralino dark matter has been analysed in the supergravity framework with assumptions of exact unification at $M_{G U T}$ in a number of papers. In particular, in Ref.[11] the neutralino relic abundance and various detection rates have been analysed in this scheme using plausible but restrictive assumptions that represent one of many possible physical scenarios

II3| J. Ellis, J. S. Hagelin, D. V. Nanopoulos and K. Tamvakis, Phys. Lett. B125 (1983) 275.

|14| M. Olechowski and S.Pokorski, Phys. Lett. B344 (1995) 201.

|15| N. Polonsky and A. Pomarol, Phys. Rev. Lett. 73 (1994) 2292; Phys. Rev. D51 (1995) 6532; D. Matalliotakis and H. P. Nilles, Nucl. Phys. B435 (1995) I15; A. Pomarol and S. Dimopoulos, CERN preprint CERN-TH/95-114 (1995); H. Murayama, Berkeley preprint LBL-36962 (1995).

|16| D. Treille, Rep. Prog. Phys. 57 (1994) 1137.

| $17 \mid$ S. Coleman and E. Weinberg, Phys. Rev. D7 (1973) 1888; G. Gamberini, G. Ridolfi and F. Zwirner, Nucl. Phys. B331 (1990) 331 ; R. Arnowitt and P. Nath, Phys. Rev. D46 (1992) 3981.

$118 \mid$ The dependence of the $\delta_{i}$ 's on the supersymmetric parameters may be evaluated explicitly, for example, in the framework of $S O(10)$ M. Olechowski (private communication, based on work with H. Murayama and S. Pokorski). 
| 19| P. Langacker and N. Polonsky, Phys. Rev. D47 (1993) 4028.

|20| Abe et al.(CDF Collaboration), Phys. Rev. D50 (1994) 2966.

121| M.S. Chanowitz, J. Ellis and M. K. Gaillard, Nucl. Phys. B128 (1977) 506; A. J. Buras, J. Ellis, M. K. Gaillard and D. V. Nanopoulos, Nucl. Phys. B135 (1978) 66.

$122 \mid$ J. Ellis. K. Enqvist, D. V. Nanopoulos and F. Zwirner, Mod. Phys. Lett. Al (1986) 57; R. Barbieri and G. Giudice, Nucl. Phys. B306 (1988) 63; M. Olechowski and S. Pokorski, Nucl. Phys. B404 (1993) 590; B. de Carlos and J. A. Casas, Phys. Lett. B309 ( 1993 ) 320; G. W. Anderson and D. J. Castaño, Phys. Rev. D52 (1995) 1693; S. Dimopoulos and G. Giudice, CERN preprint CERN-TH/95-188 (1995).

123I M. Srednicki, R. Watkins and K. Olive, Nucl. Phys. B310 (1988) 693; J. Ellis, L. Roszkowski and Z. Lalak, Phys. Lett. B245 (1990) 545: J. Ellis, J. S. Hagelin, D. V. Nanopoulos, K. Olive and M. Srednicki, Nucl. Phys. B238 (1984) 453.

|24| G. Gelmini and P. Gondolo, Nucl. Phys. B360 (1991) 145.

[25] K. Griest. M. Kamionkowski and M. S. Turner, Phys. Rev. D4] (1990) 3565; M. Drees and M. M. Nojiri, Phys. Rev. D47 (1993) 3776.

|26| A. Bottino, V. de Alfaro, N. Fornengo, G. Mignola and M. Pignone, Astroparticle Physics 2 (1994) 67.

127| F. M. Borzumati, M. Drees and M. M. Nojiri, Phys. Rev. D5I (1995) 341;

J. Wu, R. Arnowitt and P. Nath, Phys. Rev. D51 (1995) 1371;

V. Barger, M.S. Berger, P. Ohmann and R. J. N. Phillips, Phys. Rev. D51 (1995) 2438

1281 A. J. Buras, M. Misiak, M. Munz and S. Pokorski, Nucl. Phys. B424 (1994) 374; A. Ali and C. Greub, Z. Phys. C60 (1993) 423.

1291 M. S. Alam et al. (CLEO Collaboration), Phys. Rev. Lett. 74 (1995) 2885.

130| M. Carena, M. Olechowski, S. Pokorski and C. E. M. Wagner, Nucl. Phys. B426 (1994) 269; R. Rattazzi and U. Sarid, Phys. Rev. D50 (1994) 7048.

I31| V. Barger, M. S. Berger. P. Ohmann and R. J. N. Phillips, Phys. Lett. B314 (1993) 351

| 32 | B. D. Wright, Wisconsin University preprint MAD/PH/812, hep-ph/9404217 (1994); Particle Data Group, Phys. Rev D50 (1994) 117.3 .

$133 \mid$ S. Abachi et al. (D0 Collaboration), FNAL preprint FERMILAB-PUB-95/057-E D0 (1995).

134 See, for instance, J.A. Casas, A. Lleyda and C. Muñoz, Madrid University preprint FTUAM 95/11 (1995) and references quoted therein

| $35 \mid$ R. Barbieri, M. Frigeni and G. F. Giudice, Nucl. Phys. B313 (1989) 725.

136. For a summary of recent experimental activities and perspectives in this field, see, for instance: L. Mosca, Proceedings of the $29 t h$ Rencontres de Moriond: Perspectives in Particle Physics, Atomic Physics and Gravitation, Villar-sur-Ollon. Switzerland, January 1994: B. Sadoulet, TAUP93 Proceedings, Nucl. Phys. B (Proc. Suppl.) 35 (1994) I 17.

$137 \mid$ S. P. Ahlen et al., Phys. Lett. B195 (1987) 603; D. O. Caldwell et al., Phys. Rev. Lett. 61 (1988) 510; I. R. Sagdev, A. K. Drukier; D. J. Welsh, A. A. Klimenko, S. B. Osetrov, A. A. Smolnikov, TAUP93 proceedings, Nucl. Phys. B (Proc. Suppl.) 35 (I994) 175; M. L. Sarsa et al., ibidem 154.

|38| D. Reusser et al., Phys. Lett. B255 (1991) 143.

|39| M. Beck, (Heidelberg-Moscow Collaboration), TAUP93 Proceedings, Nucl. Phys. B (Proc Suppl) 35 (1994) 150.

|40| P. Belli et al. (DAMA Collaboration), Nucl. Instr. Meth. Phys. Res. A336 (1993) 336 and Proceedings of the 1995 International Cosmic Rays Conference (to appear); P. Belli and R. Bernabei, private communication.

|41| K. Griest, Phys. Rev. D38 (1988) 2357; Phys. Rev. Lett. 61 (1988) 666.

$142 \mid$ M. W. Goodman and E. Witten, Phys. Rev. D31 (1985) 3059.

$143 \mid$ S. J. Dong and K. F. Liu, Proceedings of Lattice 94, Nucl. Phys. B (Proc. Suppl.) 42 (1995) 322

$144 \mid$ T. P. Cheng. Phys. Rev. D38 (1988) 2869; H. -Y. Cheng, Phys. Lett. B219 (1989) 347.

$145 \mid$ J. Gasser, H. Leutwyler and M. E. Sainio, Phys. Lett. B253 (1991) 252.

146| I. Bijnens, I. Prades and F de Rafael, Phys. Lett. B348 (1995) 226.

147| G. Brown and M. Rho, hep-ph/9504250 (1995).

| $48 \mid$ J. Ellis and M. Karliner, Phys. Lett. B341 (1995) 397.

149| J. Engel, Phys. Lett. B264 (1991) 114.

$150 \mid$ M. T. Ressel, M. B. Auferheide, S. D. Bloom, K. Griest. G. J. Mathews and D. A. Resler, Phys. Rev. D48 (1993) 5519.

15I| E. l. Gates, G. Gyuk and M. S. Turner, FNAL preprint FERMILAB-PUB-95/090-A, (1995).

152] P. H. Chankowski and S. Pokorski, Warsaw University preprint IFT-95/5 (1995). 